\title{
UV (in)sensitivity of Higgs inflation
}

\author{
Jacopo Fumagalli and Marieke Postma \\ Nikhef, \\ Science Park 105, 1098 XG Amsterdam, The Netherlands \\ E-mail: jacopof@nikhef.nl, mpostma@nikhef.nl
}

ABSTRACT: The predictions of Standard Model Higgs inflation are in excellent agreement with the Planck data, without the need for new fields. However, consistency of the theory requires the presence of (unknown) threshold corrections. These modify the running of the couplings, and thereby change the shape of the inflationary potential. This raises the question how sensitive the CMB parameters are to the UV completion. We show that, due to a precise cancellation, the inflationary predictions are almost unaffected. This implies in general that one cannot relate the spectral index and tensor-to-scalar ratio to the precise top and Higgs mass measurements at the LHC, nor can one probe effects of UV physics on the running.

Keywords: Cosmology of Theories beyond the SM, Higgs Physics, Renormalization Group, Effective field theories

ARXIV EPRINT: 1602.07234 


\section{Contents}

1 Introduction 1

2 Effective action for Higgs inflation $\quad 3$

2.1 Higgs inflation in Einstein frame 3

2.2 UV completion and threshold corrections 4

$\begin{array}{lll}2.3 & \text { Renormalization prescription } & 6\end{array}$

2.4 Renormalization group equations 9

$\begin{array}{llr}3 & \text { Inflation } & \mathbf{1 1}\end{array}$

3.1 Inflation on the flat plateau 11

$\begin{array}{ll}3.2 & \text { Inflation near the maximum } \\ \end{array}$

4 Conclusions $\quad 19$

$\begin{array}{ll}\text { A Effective action and renormalization group improvement } & \mathbf{2 0}\end{array}$

$\begin{array}{lll}\text { A.1 Canonical kinetic sector } & 21\end{array}$

A.2 Non canonical kinetic sector 23

B CMB parameters at higher order in $\delta \quad 24$

\section{Introduction}

Standard Model Higgs inflation [1-4] has attracted much attention over the last years. This is not surprising, as the model has many appealing features - at least, at the classical level. With the Higgs field as inflaton the model is firmly embedded in the Standard Model (SM). Only one new interaction is needed, a non-minimal coupling of the Higgs field to the Ricci tensor, making the set-up minimal. And finally, the predictions for the inflationary observables are in excellent agreement with the latest Planck data [5]

Although this minimal approach is attractive, it is not clear whether Higgs inflation is fully consistent as a quantum theory. First, including the running of couplings, the potential may become unstable at energy scales below the inflationary scale. For the best fit values of the top and Higgs mass this indeed happens, but it should be noted that vacuum stability of the SM up to the Planck scale is only excluded at the $2-3 \sigma$ level [6-12]. Furthermore, with extra matter, e.g. a dark matter particle, the instability bound can be evaded [13-15].

A second issue with quantum Higgs inflation is the unitarity bound [16-23]. Tree level unitarity is lost at energies well below the Planck scale, and new degrees of freedom $[24,25]$ or strong dynamics [26, 27]) should become important at this scale. Although 
the energy scale of the inflationary potential is always below the field-dependent unitarity cutoff $[20,21,28,29]$, which makes the semiclassical approximation meaningful, this is not so for the field value. To forbid non-renormalizable operators that spoil the inflationary potential already at the classical level, an (approximate) shift symmetry has to be assumed. This is no different from chaotic inflation.

Thirdly, the theory is not renormalizable over the full field range. It has been shown that for small, mid and large field values Higgs inflation is renormalizable in the usual effective field theory (EFT) sense [30] (see also earlier work [31-39]). However, these EFTs need to be patched together at the boundaries of the different field regimes, and it is here that we expect non-renormalizable operators to become important. We will also refer to these higher order operators as threshold corrections, and more generically, speak about threshold corrections to the renormalization group equations (RGEs) or to the inflationary observables, meaning the effect of the higher order operators on these quantities.

Thus for a consistent quantum field theory, new physics is needed below the Planck scale. This begs the question: how sensitive is Higgs inflation to the unknown UV physics $[22,40]$ ? If the model predictions demanded a particular UV completion it would mean that the simplicity of the set-up, to which it owes much of its success, would be completely spoiled. In this paper we will show that as long as the UV corrections do not affect the inflaton potential at tree level, but only enter at loop level via corrections to the renormalization group equations, the inflationary predictions are to a very good approximation unaffected. Indeed, whatever the exact running of the couplings, the spectral index $n_{s}$ and tensor-to-scalar ratio $r$ have at leading order in the slow roll expansion a universal value: ${ }^{1}$

$$
n_{s}=1-\frac{2}{N_{\star}}+\mathcal{O}\left(N_{\star}^{-2}\right) \simeq 0.967, \quad r=\frac{12}{N_{\star}^{2}}+\mathcal{O}\left(N_{\star}^{-3}, \xi^{-1}\right) \simeq 0.003
$$

with $N_{\star} \approx 60$ the number of efolds of observable inflation, and $\xi \gg 1$ the non-minimal coupling. We can rephrase our statement as follows: as long as the non-minimally coupled Higgs is a viable inflaton candidate - no large tree-level UV corrections to the potential, and no RGE induced instability of the potential - the predictions are extremely robust and to high precision are equal to the tree level results, in excellent agreement with the Planck data on the CMB [5].

This paper is organized as follows. In the next section we first describe the full quantum action for Higgs inflation. To set the notation, in section 2.1 we introduce the classical action. In section 2.2 we discuss the UV completion of the theory. Following [22] we introduce a set of threshold corrections, which we will use in our numerical results. The particular set of non-renormalizable operators can be motivated in two ways, either by assuming an approximate shift symmetry, or by demanding that UV physics only enters where needed for the consistency of the theory, namely at the boundary of the small and

\footnotetext{
${ }^{1}$ This is for inflation on the flat plateau of the potential, as is usually meant by "Higgs inflation" (and at tree level is the only possibility). For some fine-tuned values of the couplings, inflation near a maximum or inflection point of the potential is possible; in this latter case, the predictions are sensitive to the details of the potential, and thus to the unknown UV physics.
} 
mid field regimes. We stress, however, that the universal results for $n_{s}$ and $r$ as given in (1.1) do not depend on this choice. There has been some debate in the literature on frame dependence and the choice of renormalization scale [31-37]. In section 2.3 we will argue that this choice is unambiguously defined [38, 41]. To end this section, in 2.4 we shortly discuss the renormalization group equations (RGEs) and also give details on the numerical implementation.

In section 3 we then turn to the predictions for inflation. In 3.1 we calculate the inflationary observables for Higgs inflation, using the RGE improved potential which includes the effects of running couplings. We show analytically that the spectral index and scalarto-tensor ratio are to first order in an $1 / N_{\star}$ expansion insensitive to the running of the couplings. To investigate the possibility of hilltop inflation, inflation near a maximum of the potential, we turn to our numerical results. As discussed in section 3.2, we find that for fine-tuned boundary conditions (the top/Higgs mass values at the electroweak scale, and the Wilson coefficients of the non-renormalizable operators) hilltop inflation is possible. Since the potential near the maximum is tuned to be flat enough for 60 efolds of inflation, it comes as no surprise that this tuning depends very sensitively on the details of the potential, and thus also on the running of the couplings.

We end in 4 with some concluding remarks.

Our sign convention for the metric is mostly positive $(-,+,+,+)$. The dependence on the Planck mass is kept explicitly only in the first part where we introduce the model and discuss the unitary cutoff, in the rest of the paper we set the reduced Planck mass to unity $m_{\mathrm{P}}=(\sqrt{8 \pi G})^{-1}=1$.

\section{Effective action for Higgs inflation}

In this section we discuss the effective action for Higgs inflation; in section 3 we then calculate the inflationary observables for this action.

The loop corrections can be incorporated in an RGE improved action with running couplings. We include a class of threshold corrections (coming from the UV completion) which only enter the inflationary physics via their effect on the renormalization group equations (RGEs). Finally, we discuss the choice of renormalization scale.

\subsection{Higgs inflation in Einstein frame}

To set the notation, let's start with defining the classical action for Higgs inflation in the Jordan frame:

$$
\mathcal{L}_{J}=\sqrt{-g^{J}}\left[\frac{1}{2} m_{\mathrm{P}}^{2}\left(1+\frac{2 \xi \Phi^{\dagger} \Phi}{m_{\mathrm{P}}^{2}}\right) R\left[g^{J}\right]+\mathcal{L}_{\mathrm{SM}}\right] .
$$

with $\Phi$ the standard model Higgs doublet and $\xi$ the non-minimal coupling to gravity. The gravitational action can be brought in Einstein-Hilbert form by a conformal transformation $g_{\mu \nu}=\Omega^{2} g_{\mu \nu}^{J}$ with conformal factor

$$
\Omega^{2}=\left(1+\frac{2 \xi \Phi^{\dagger} \Phi}{m_{\mathrm{P}}^{2}}\right)
$$


The resulting Einstein frame action is

$$
\mathcal{L}_{E}=\sqrt{-g}\left[\frac{1}{2} m_{\mathrm{P}}^{2} R[g]-\frac{1}{\Omega^{2}}\left(\partial_{\mu} \Phi\right)^{\dagger}\left(\partial^{\mu} \Phi\right)-\frac{3 \xi^{2}}{m_{\mathrm{P}}^{2} \Omega^{4}} \partial_{\mu}\left(\Phi^{\dagger} \Phi\right) \partial^{\mu}\left(\Phi^{\dagger} \Phi\right)-\frac{V_{J}}{\Omega^{4}}+\ldots\right],
$$

with $V_{J}=\lambda\left(\Phi^{\dagger} \Phi-v^{2} / 2\right)^{2}$. The Lagrangian for the classical background field $\Phi=\frac{1}{\sqrt{2}}\left(\begin{array}{l}0 \\ \phi\end{array}\right)$ is

$$
\mathcal{L}_{E}=\sqrt{-g}\left[\frac{1}{2} m_{\mathrm{P}}^{2} R[g]-\frac{1}{2} \gamma_{\phi \phi}(\phi)(\partial \phi)^{2}-\frac{\lambda\left(\phi^{2}-v^{2}\right)^{2}}{4 \Omega^{4}}\right] .
$$

In the large field limit $\phi^{2} \gg m_{\mathrm{P}}^{2} / \xi$ the potential approaches a constant value developing a flat plateau that can support a period of slow roll inflation. The classical Higgs field can be canonically normalized via

$$
\frac{1}{2} \gamma_{\phi \phi}(\phi)(\partial \phi)^{2}=\frac{1}{2 \Omega^{2}}\left(1+\frac{6 \xi^{2}}{m_{\mathrm{P}}^{2} \Omega^{2}} \phi^{2}\right)(\partial \phi)^{2}=\frac{1}{2}(\partial h)^{2},
$$

with $\Omega^{2}(\phi)=1+\xi \phi^{2} / m_{\mathrm{P}}^{2}$ evaluated on the classical background.

The $v^{2}$-term in the Higgs potential plays no role during inflation, and we set it to zero in the following.

\subsection{UV completion and threshold corrections}

We only consider higher order operators that change the inflationary potential at loop level. This can be motivated independently in two ways. Either assume that the UV completion respects an approximate shift symmetry, which forbids the most dangerous operators that already change the potential at tree level. Or adopt a minimal approach to UV corrections, only adding higher dimensional operators that are really necessary for consistency of the theory. The result in both cases is that the unknown UV physics only enters the inflationary potential via their effect on the renormalization group equations and thus on the running of the couplings. As we will show analytically in the next section, the inflationary predictions are universal, and all dependence on the running, and thus on the threshold corrections, drops out.

Below we will motivate our choice of higher dimensional operators that we add, and that we will use in our numerical computations. We would like to stress, though, that this choice is not critical to our results, and other parameterizations can be chosen and additional corrections can be added. As long as inflation is possible at all - no large corrections to the tree level potential and UV physics only enters via the RGE equationsthe inflationary predictions are robust.

It is well known that for a large non-minimal coupling $\xi \gg 1$, as needed for Higgs inflation, unitarity of tree level scattering breaks down well below the Planck scale. The unitarity cutoff - the momentum scale at which tree-level unitarity is violated - depends on the field value of the Higgs field, and is given by [20-22]:

$$
\Lambda \sim\left\{\frac{m_{\mathrm{P}}}{\xi}, \phi, \frac{m_{\mathrm{P}}}{\sqrt{\xi}}\right\}
$$


in respectively the small, mid, and large field regimes, defined via

$$
\text { small field : } \phi<\frac{m_{\mathrm{P}}}{\xi}, \quad \text { mid field : } \frac{m_{\mathrm{P}}}{\xi}<\phi<\frac{m_{\mathrm{P}}}{\sqrt{\xi}}, \quad \text { large field : } \frac{m_{\mathrm{P}}}{\sqrt{\xi}}<\phi .
$$

The field dependence of the cutoff may be understood from integrating out heavy fields with a field dependent mass. In the case that the cutoff signals the onset of strong dynamics, the field dependence also may arise naturally.

Over the whole field region the typical energy in the Higgs potential is below the cutoff $V(\phi)^{1 / 4}<\Lambda(\phi)$. Nevertheless, the field value during inflation exceeds the unitarity cutoff. Following the usual effective field theory approach, and adding all operators that respect the symmetries of theory, the model is extremely sensitive to UV physics. Indeed, all higher operators of the form $\left(\Phi^{\dagger} \Phi\right)^{n+2} / \Lambda^{n}$ will completely spoil the inflationary potential. Also operators of the form $\left(\Phi^{\dagger} \Phi\right)^{n} \mathcal{O}^{4} / \Lambda^{n}$ should be forbidden during inflation; here $\mathcal{O}^{4}$ is a dimension four operator made up of standard model fields, e.g. $\mathcal{O}^{4}=F^{\mu \nu} F_{\mu \nu}$ and $F_{\mu \nu}$ the $\mathrm{SU}(2)$ field strength tensor. Indeed, during inflation these operators will give rise to effective couplings that are non-perturbatively large, and thus spoil the predictiveness of the model. In this sense the situation in Higgs inflation is not different from chaotic models of inflation. In the latter case the cutoff is the Planck scale and inflation takes place at superplanckian field values, and thus also chaotic inflation is highly sensitive to operators of the above form.

To avoid the dangerous operators discussed in the previous paragraph, we assume that the UV completion respects the approximate shift symmetry of the action in the inflationary regime, which is only broken by a non-zero (but small) Higgs mass. This implies that at dimension six, which are the leading corrections, only operators of the form $[22]^{2}$

$$
\mathcal{L} \supset \sum_{i} c_{i} \frac{m_{h}^{2}}{\Lambda^{2}} \mathcal{O}_{i}^{4}
$$

are allowed. Here $c_{i}$ are unknown Wilson coefficients, and the sum is over all dimension four operators invariant under the SM symmetries. The cutoff is chosen as the field dependent unitarity bound, which is an additional (but natural) assumption. Using the explicit form of the Higgs mass (see (2.10) below) and unitarity bound (2.6), it can be seen that these operators are only unsuppressed around the scale (we set $m_{\mathrm{P}}=1$ from now on) $\phi \sim 1 / \xi$. As a result, operators of the form (2.8) do not affect the tree level inflaton potential. They can, however, affect the inflationary potential at the quantum level, as these operators give corrections to the RGE equations [22, 42]. Running the SM couplings from the electroweak scale, where they are measured, to the inflationary scale, one has to pass the region where the normalization scale is $\mu \sim 1 / \xi$ and the threshold corrections - if large enough cannot be neglected.

We can arrive at the same conclusion, i.e. threshold corrections that are important only at $\phi \sim 1 / \xi$, from a different perspective. Namely: take the minimalistic approach to add new physics only when really necessary for the consistency of the theory. For this

\footnotetext{
${ }^{2}$ In the small field regime $m_{h}^{2} \propto H^{\dagger} H$ and the operators (2.8) reduce to the six dimensional operators listed in [42].
} 
purpose we do not need, for the reasons we are going to explain in a moment, higher dimensional operators that become important at the large field values during inflation, only corrections around the scale $\phi \sim 1 / \xi$ are necessary. First of all, although $V^{1 / 4}<\Lambda$ at all field values, these scales become of the same order at $\phi \sim 1 / \xi$ and corrections to the Higgs inflation action are unsuppressed. Secondly, the counterterms introduced to absorb the UV divergencies of the quantum corrections make a jump at the scale $\phi \sim 1 / \xi[30,43]$, which signals that new physics should enter at this scale. Let us explain this second point in more detail.

The one-loop effective potential for the classical field $\phi$ is given by the tree level potential plus the Coleman-Weinberg potential [44]. Explicitly

$$
V_{\text {eff }}=V_{\text {tree }}+\frac{1}{64 \pi^{2}} \sum_{i}(-1)^{F_{i}} S_{i} m_{i}^{4}(\phi)\left[\ln \left(\frac{m_{i}^{2}(\phi)}{\mu^{2}}\right)-c_{i}\right]
$$

in the $\overline{\mathrm{MS}}$ renormalization scheme. Here $\mu$ is the normalization scale, $F_{i}=0$ (1) for a boson (fermion) field, $S_{i}$ counts the degrees of freedom of each particle with mass $m_{i}$, and $c_{i}=3 / 2$ for fermions and scalars and $c_{i}=5 / 6$ for gauge bosons. The gauge boson, top quark, Higgs and (three) Goldstone boson (GB) masses are given (in Landau gauge) by [30, 43]

$$
m_{A^{i}}^{2}=\frac{g_{i}^{2} \phi^{2}}{2 \Omega^{2}}, \quad m_{t}^{2}=\frac{y_{t}^{2} \phi^{2}}{2 \Omega^{2}}, \quad m_{h}^{2}=3 \lambda \phi^{2} \frac{1+4 \xi^{2} \phi^{2}-4 \xi^{3} \phi^{4}}{\Omega^{4}\left(1+6 \xi^{2} \phi^{3}\right)^{2}}, \quad m_{\theta^{i}}^{2}=\frac{\lambda \phi^{2}}{\Omega^{4}\left(1+6 \xi^{2} \phi^{2}\right)},
$$

with $g_{i}=\left\{g_{2}, \sqrt{g_{1}^{2}+g_{2}^{2}}\right\}$ for the $W$ and $Z$ bosons with $g_{1}, g_{2}$ the hypercharge $\mathrm{U}(1)_{Y}$ and and $\mathrm{SU}(2)$ couplings respectively, and $y_{t}$ the top Yukawa. The loop contribution from the gauge bosons and top quark has the same field dependence as the tree level potential, and the divergencies can be absorbed in the whole field range. However, that is not the case for the Higgs and GB masses. The theory is not renormalizable over the full field range. It has been shown in [30] that nevertheless in the small, mid and large field regimes (2.7) a renormalizable EFT can be constructed. That is, when the Lagrangian is expanded in a small parameter that defines the given regime, all divergencies can be absorbed order by order in a finite number of counter terms; no new operators beyond those already present in the tree level Lagrangian are needed. The EFTs are valid only within the given regime, and for energies below the (field-dependent) unitarity cutoff (2.6). The renormalization group equations in the small field regime are those of the Standard Model. The RGEs in the mid and large field regimes are the same, and differ from the SM RGEs because of the non-minimal coupling. The EFTs need to be patched together at the boundaries. As is clear, at least at the border between the small and mid field regime, which is at $\phi \sim 1 / \xi$, threshold corrections are needed as it is here that the RG equations change.

\subsection{Renormalization prescription}

Higgs inflation can be analyzed, and loop corrections can be calculated in both the Jordan (2.1) and Einstein frame (2.4). Even if the frames are merely related by a field transformation it is not universally accepted that they describe the same physics. The equivalence of the Jordan and Einstein frame [45-47] can be made explicit by rewriting the action 
in terms of dimensionless quantities which are invariant under a conformal transformation $[41,48]$. The equivalence can also be checked on a case-by-case basis. For example, in [49-53] it was shown that both frames give the same result for the curvature perturbation during inflation, [54] uses a covariant approach to show that both frames gives the same (on-shell) effective action, and in [28] the same covariant approach was used to show that the RGE equation for $\xi$ is the same in both frames. Finally, in [30] it was shown that the Coleman-Weinberg potential and the renormalization procedure is one-to-one in both frames.

Despite all this there remains confusion in the literature on the frame dependence of the results, and in particular on the choice of renormalization scale. Here we review that if the renormalization prescription is done carefully no such ambiguity arises, more details can be found in [30]. Another way to arrive at the same conclusion is to do the one-loop analysis using dimensionless quantities invariant under a conformal transformation, the approach advocated in $[41,48]$ (which trivially corresponds to the Einstein frame analysis).

To include the (one-loop) correction one could proceed in two ways: ${ }^{3}$

1. First go to the Einstein frame and then add the quantum correction to $V_{E}$.

$$
V_{E_{1}}=V_{E}^{(0)}(\phi)+V_{E}^{(1)}=\frac{V_{J}^{(0)}}{\Omega^{4}}+V_{E}^{(1)}
$$

where the superscript (0) and (1) refer to the tree level and one-loop ColemanWeinberg potential respectively.

2. Add the CW corrections to the Jordan frame potential and only after transform to the Einstein frame

$$
V_{J}^{(0)}(\phi)+V_{J}^{(1)} \stackrel{E}{\longrightarrow} V_{E_{2}}=\frac{V_{J}^{(0)}(\phi)}{\Omega^{4}}+\frac{V_{J}^{(1)}}{\Omega^{4}} .
$$

As can be seen in the above equations, but this is general, all mass scales are rescaled by the conformal transformation

$$
m_{J} \stackrel{E}{\longrightarrow} m_{E}=\frac{m_{J}}{\Omega} .
$$

If one does not consider the back reaction from gravity, following one of the two paths leads to different results in the Higgs-gravity sector. This is understandable since degrees of freedom considered frozen in one frame are dynamical in the other and vice versa. ${ }^{4}$ Since the main contribution to the $\mathrm{CW}$ potential comes from the top quark and gauge boson loops - the Higgs and GB loops are suppressed - we do not have to worry about this.

Let's consider then the contribution of the top quark to the Coleman-Weinberg potential in the Einstein frame, following procedure 1

$$
V_{E_{1}}=\frac{(\lambda+\delta \lambda) \phi^{4}}{4 \Omega^{4}}+\frac{1}{8 \pi^{2}} m_{t, E}^{4} \ln \left(\frac{\Lambda_{E}^{2}}{m_{t, E}^{2}}\right),
$$

\footnotetext{
${ }^{3}$ In both cases we want to end up in the Einstein frame where slow roll inflation is most easily studied.

${ }^{4}$ For example, the Sasaki-Mukhanov variable is a different combination of the scalar metric degree of freedom and the Higgs in the two frames. To leading order in the slow roll approximation, one can treat gravity classically in the Einstein frame as the effects from back reaction are suppressed [30]; however, this is not the case for the Jordan frame, and care should be taken when considering the Higgs and GB loops.
} 
where cutoff regularization has been used; $\delta \lambda$ is the counter term, and the Einstein frame top mass has been defined in $(2.10)$. Choosing the counterterm $\delta \lambda=-\frac{y^{4}}{\left(4 \pi^{2}\right)} \ln \left(\frac{\Lambda_{E}^{2}}{\mu_{E}^{2}}\right)$ gives

$$
V_{E_{1}}=\frac{\phi^{4}}{4 \Omega^{4}}\left(\lambda+\frac{y^{4}}{8 \pi^{2}} \ln \left(\frac{\mu_{E}^{2}}{m_{t, E}^{2}}\right)\right) .
$$

The $\log$ will be minimized for $\mu_{E}=m_{t, E}$; in the RG improved action this will then minimize the error, see appendix A. This choice of renormalization scale is often referred to as "prescription 1". For Higgs inflation it corresponds to the usual prescription that the renormalization scale is chosen to be the typical energy scale involved in the process.

Procedure 2 gives

$$
V_{J}=\frac{(\lambda+\delta \lambda) \phi^{4}}{4}+\frac{1}{8 \pi^{2}} m_{t, J}^{4} \ln \left(\frac{\Lambda_{J}^{2}}{m_{t, J}^{2}}\right)=\frac{\lambda \phi^{4}}{4}+\frac{1}{8 \pi^{2}} m_{t, J}^{4} \ln \left(\frac{\mu_{J}^{2}}{m_{t, J}^{2}}\right)
$$

where $m_{t, J}=\Omega m_{t, E}$ is the top mass in the Jordan frame. In the second expression we set the counterterm $\delta \lambda=-\frac{y^{4}}{4 \pi^{2}} \ln \left(\frac{\Lambda_{J}^{2}}{\mu_{J}^{2}}\right)$. At this stage the log in the potential will be minimized for $\mu_{J}(t) \sim m_{t, J}$. This choice is often referred to as "prescription 2". However, this expression is still in Jordan frame units. Expressing the renormalization scale in Planck units

$$
\frac{\mu_{J}}{m_{\mathrm{pl}, J}}=\frac{m_{t, J}}{m_{\mathrm{pl}, J}}=\frac{m_{t, E}}{m_{\mathrm{pl}, E}}=\frac{\mu_{E}}{m_{\mathrm{pl}, E}},
$$

we see it is exactly the same prescription as in the Einstein frame. Here it should be noted that all mass scales, including the Planck mass, cutoff scale and renormalization scale, are rescaled as in (2.13) under a conformal transformation. ${ }^{5}$ Finally, transforming the Jordan frame potential to the Einstein frame we retrieve $V_{E_{2}}=V_{E_{1}}$. There is no ambiguity in the renormalization scale, which is correctly given by prescription 1. For definiteness, we will use in the next section

$$
\mu_{E}=\frac{\phi}{\Omega(\phi, \xi)} .
$$

Although different renormalization prescriptions do not arise from frame dependence, one could still argue that they encode different UV completions of the theory. As discussed around (2.9) no counterterms can be defined that absorb the (subdominant) Higgs and GB contributions over the whole field range. Consider then field dependent counterterms of the form $\delta \lambda=-\frac{y^{4}}{4 \pi^{2}} \ln \left(\frac{\Lambda^{2}}{\mu^{2}} f(\phi)\right)$. The Einstein frame potential becomes

$$
V_{E}=\frac{\lambda \phi^{4}}{4 \Omega^{4}}+\frac{1}{8 \pi^{2}} m_{t}^{4} \ln \left(\frac{m_{t}^{2}}{\mu^{2}} f(\phi)\right) .
$$

\footnotetext{
${ }^{5}$ The situation is completely analogue to going from a conformal FLRW metric to a Minkowksi metric by doing a conformal transformation with $\Omega=a(t)$ the scale factor. All masses are rescaled by the scale factor, cf. the physical momentum (the canonical momentum in the FLRW metric) and comoving momentum (the canonical momentum in the Minkowski metric) are related by $k_{\mathrm{com}}=k_{\mathrm{phys}} / a$. Spurious factors of $a(t)$ (the scale factor is by definition unobservable) only appear when comoving scales are erroneously compared to physical mass scales [55].
} 
The choice $f(\phi)=\Omega^{2}$ corresponds to prescription 2 , as $\mu=f(\phi) m_{t}$ will minimize the log. Note however, that a field dependent counterterm implies adding new operators to the action. Indeed the above expression could only have come from a potential of the form

$$
V_{\mathrm{eff}}=\frac{(\lambda+\delta \lambda)}{4 \Omega^{4}} \phi^{4}+\frac{1}{8 \pi^{2}} m_{\tau}^{4} \ln \left(\frac{\Lambda^{2}}{m_{\tau}^{2}}\right)+\frac{1}{8 \pi^{2}} m_{\tau}^{4} \ln (f(\phi)) .
$$

Therefore, a non-trivial choice of $f(\phi)$ implies that the potential is already changed at the classical level! The simple form of the action in the Jordan frame (2.1), with just a single new parameter compared to the SM Lagrangian, can no longer be used as a motivation for the model. Moreover, allowing for any UV completion possible, i.e. for any choice of $f(\phi)$, all predictivity is lost as literally any potential can be constructed. Fortunately, that is not needed. The choice $f(\phi)=1$ is the natural choice as no new operators and counterterms beyond those present in standard Higgs inflation (2.4) are needed in the large field regime. As has been shown in [30] for $f(\phi)=1$ a renormalizable EFT can be constructed in the small, mid and large field regime. On the boundaries of these regimes, and as discussed in section 2.2, especially between the small and mid field regime, threshold corrections are needed. But for consistency alone, adding new corrections in the large field regime is not demanded.

Let us stress a crucial point about this way of parametrizing the renormalization scale. We have already seen that the cutoff depends on the Higgs vev. In the low field regime $\Lambda_{\text {low field }} \equiv 1 / \xi$. If one is interested in the RG flow at energy scales beyond $1 / \xi$ it might seem it is not possible to say anything without knowing exactly the form of the UV completion. Consider the analogy with the Fermi effective theory of beta decay. At energy values below the $W$-boson mass the Fermi effective action can be used to compute the beta functions etc. However, for energies above the cutoff ( $W$ mass) the knowledge of the full electroweak Lagrangian is needed. The situation here is considerably different. The "prescription 1" choice of the renormalization scale automatically takes in account that when $\mu(\phi)>\Lambda_{\text {low field }}$ the field is no longer in the low field region and the unitary bound is still larger than $\mu .{ }^{6}$ Thus the full form of the UV completion is not needed and one can consistently parametrize it with a series of higher order operators suppressed by the scale given by the field dependent cutoff. ${ }^{7}$ From the physical point of view the difference between the two situations can be understood from the fact that here the increase in energy is due to a displacement of the Higgs vev.

\subsection{Renormalization group equations}

In the next section we will calculate the inflationary observables taking into account the running of all couplings. In particular, we consider the RG improved action (2.4) with

\footnotetext{
${ }^{6}$ Prescription 1 gives $\mu(\phi)<\Lambda(\phi)$ for all field values. For prescription 2 , on the other hand $\mu(\phi)>\Lambda(\phi)$ for large field values, and the RGE evolution can no longer be described in the EFT setting, the full UV completion is required.

${ }^{7}$ If a constant cutoff $\Lambda_{\text {low field }}$ is chosen you would need for instance an extra degree of freedom to restore the unitarity of the model till the Planck scale [24, 25]. These UV completions modify the inflaton potential already at tree level, and thus they are different from the ones discussed in this paper.
} 
potential and field space metric (from now on we set $V_{\text {eff }} \equiv V$ )

$$
V=\frac{\lambda(t) \phi^{4}}{4\left(1+\xi(t) \phi^{2}\right)^{2}}, \quad \gamma=\frac{1+\xi(t) \phi^{2}(1+6 \xi(t))}{\left(1+\xi(t) \phi^{2}\right)^{2}},
$$

with

$$
t=\ln \left(\mu / m_{\mathrm{t}}\right)
$$

and $m_{t}$ the EW scale top mass. In addition, the renormalization scale (2.18) depends on the running couplings

$$
\mu=\frac{\phi}{\sqrt{\left(1+\xi(t) \phi^{2}\right)}} .
$$

For more details see the discussion in appendix A.

The running of the couplings is governed by the RG equations. In the small field regime these are just the SM RGEs, we use the two-loop result and the EW boundary conditions defined in $[37,56]$. The one-loop RGEs for the mid and large field regime have been derived by different groups [31-39], with small differences. We use the recent result of [30]. Our main conclusions will not depend on this choice, only the exact numerical values of parameters might differ slightly. We set the boundary condition for $\xi_{0}$ at the boundary of the mid-field regime $\xi(1 / \xi)=\xi_{0}$.

In $[22,42]$ the corrections to the $\beta$ functions due to the higher dimensional operators (2.8) were calculated. The corrections depend on unknown Wilson coefficients $c_{i}$, the Higgs mass which is given in (2.10), and the cutoff scale that we choose

$$
\Lambda=\frac{\left(1+\xi(t)^{2} \phi^{2}\right)}{\xi(t)^{2}\left(1+\xi(t) \phi^{2}\right)},
$$

which reduces to the unitarity cutoff in the three regimes (2.6). Since the operators are peaked at $1 / \xi$, only around this scale the corrections to the running are appreciable. For inflationary purposes the effect is that threshold corrections may give a "kick" to $\lambda$, i.e. change $\lambda(\mu \sim 1 / \xi)$ by some amount compared to the SM running. Since $\lambda \ll 1$ this kick may be appreciable for Wilson coefficients $c_{i} \sim \mathcal{O}(10)$ (such that the threshold and SM contribution to the RGEs are of comparable size $\delta \beta \sim \beta_{\mathrm{SM}}$ at the scale $\left.\mu=1 / \xi\right)$. The relative kick to other SM parameters is very small. For our numerical results we choose the Wilson coefficients, defined in the appendix B of [22], randomly in the interval

$$
c_{i}=\operatorname{Random}\left[-c^{\max }, c^{\max }\right] .
$$

In our numerics, we choose boundary conditions at the EW scale, $\xi_{0}$ at the intermediate scale, and a set of Wilson coefficients $c_{i}$, and then run all couplings to the large field regime. We then determine $t_{\text {end }}$ and $t_{\star}$, i.e. the normalization scale (2.22) at the end and $N_{\star}$ efolds before the end of inflation, and finally the power spectrum for the perturbations. We reiterate this procedure, adjusting the value of $\xi_{0}$ till the right COBE normalization (3.17) is obtained. It may happen that for some or all $\xi_{0}$-values inflation with more than $N_{\star}$ efolds is impossible. For definiteness, we take $N_{\star}=60$. In the next section we discuss the calculation of the perturbations during inflation in detail. 


\section{Inflation}

In this section we compute the spectral index and tensor-to-scalar ratio taking into account the running of the couplings. We will show analytically in the next subsection that for inflation on a flat plateau, as it is usually assumed in Higgs inflation, all dependence on the beta-functions drops out, and the inflationary observables are the same as for the classical potential. With running included, it is possible for a limited range of parameters that the potential develops a maximum. As discussed in subsection 3.2 for hilltop inflation, i.e. inflation near the maximum, the results depend sensitively on the running, and thus on the UV completion (entering via the beta-functions). We present numerical results for this case.

\subsection{Inflation on the flat plateau}

Higgs inflation takes place in the large field regime (2.7), where the action can be expanded in the small parameter

$$
\delta=1 /\left(\xi \phi^{2}\right) \ll 1 .
$$

As follows from (3.7), (3.13) below, the $\delta$-expansion is equivalent to an expansion in slow roll parameters, and is also equivalent to an $1 / N_{\star}$ expansion.

In order to include the effects of running couplings on the inflationary observables we work with the renormalization group improved action. The potential and field space metric (2.21) can be rewritten as

$$
V=\frac{\lambda(t)}{4 \xi(t)^{2}} \frac{1}{(1+\delta(t))^{2}}, \quad \gamma_{\phi \phi}=\frac{\delta(t)(1+\delta(t)+6 \xi(t))}{(1+\delta(t))^{2}}, \quad \delta(t)=\frac{1}{\xi(t) \phi^{2}},
$$

with $t=\ln \left(\mu / m_{t}\right), m_{t}$ the EW scale top mass, and $\mu$ the renormalization scale (2.23)

$$
\mu=\frac{1}{\sqrt{\xi(t)(1+\delta(t))}}
$$

which is proportional to the top and gauge boson mass. This choice minimizes the logs in the Coleman-Weinberg expansion, as already discussed in section 2.3. For $\delta \ll 1$ the potential reduces to a constant plus (exponentially) small corrections, and inflation takes place on a flat plateau. The running of the couplings may slightly tilt the plateau, and thus change the expressions for the observables.

To calculate the slow roll parameters the first and second derivatives of the potential with respect to the canonically normalized field $h$, defined in (2.5), are needed. Let's start with the slope first. Using the chain rule gives

$$
V_{h}=\frac{1}{\sqrt{\gamma_{\phi \phi}}}\left(\frac{\partial V}{\partial \phi}+\frac{\partial V}{\partial \lambda} \lambda_{\phi}+\frac{\partial V}{\partial \xi} \xi_{\phi}\right)
$$

with

$$
\lambda_{\phi}=\beta_{\lambda} \frac{d t}{d \phi}, \quad \xi_{\phi}=\beta_{\xi} \frac{d t}{d \phi}, \quad \frac{d t}{d \phi}=\frac{\delta^{3 / 2} \xi^{1 / 2}}{1+\delta+\frac{\beta_{\xi}}{2 \xi}}
$$


where we used the definitions $\beta_{\lambda}=\partial \lambda / \partial t$ and $\beta_{\xi}=\partial \xi / \partial t$. The last expresson follows from the explicit form of normalization scale (3.3). Putting it all together gives

$$
\frac{V_{h}}{V}=\sqrt{\frac{8}{3}} \frac{\delta(1+\delta)}{\sqrt{1+\frac{(1+\delta)}{6 \xi}}} \frac{\left(1+\frac{\beta_{\lambda}}{4 \lambda}\right)}{\left(1+\delta+\frac{\beta_{\xi}}{2 \xi}\right)} .
$$

This result is still exact, no $\delta$-expansion or other approximation has been done. In a similar way the 2 nd derivative of the potential can be computed. The slow roll parameters become

$$
\epsilon \equiv \frac{1}{2}\left(\frac{V_{h}}{V}\right)^{2}=\frac{4}{3} \delta^{2} F^{2}\left(1+\frac{1}{6 \xi}\right)+\mathcal{O}\left(\delta^{3}\right), \quad \eta \equiv \frac{V_{h h}}{V}=-\frac{4}{3} \delta F+\mathcal{O}\left(\delta^{2}\right),
$$

with

$$
F=\frac{\left(1+\frac{1}{4} \frac{\beta_{\lambda}}{\lambda}\right)}{\left(1+\frac{1}{2} \frac{\beta_{\xi}}{\xi}\right)\left(1+\frac{1}{6 \xi}\right)}
$$

Turning off the running of the couplings $\beta_{\lambda}=\beta_{\xi}=0$, we retrieve the standard classical results (which are often expressed in the large $\xi$ limit where $F=1+\mathcal{O}(1 / \xi)$ ).

The RGEs for SM Higgs inflation have been calculated in the literature. In the large field regime they differ from the SM ones; we quote the recent results [30]

$$
\frac{\beta_{\lambda}}{\lambda}=\frac{1}{8 \pi^{2}}\left(\frac{3 g^{4}-y^{4}}{\lambda}-2 y^{2}\right), \quad \frac{\beta_{\xi}}{\xi}=\frac{1}{8 \pi^{2}} y^{2} .
$$

Note that in the inflationary regime the contribution from threshold corrections to the beta functions can be neglected since for our choice their contribution is important only around the scale $\mu=1 / \xi$. The main point is that $\beta_{\xi} / \xi<1$ is always perturbatively small and the denominator of $F$ is always positive. The top contribution dominates and $\beta_{\lambda}<0$ at the inflationary scale. This means that $F$ can go through zero and become large and negative in the $\lambda \rightarrow 0$ limit. When

$$
F=0 \quad \Leftrightarrow \quad \lambda_{\max }=-\frac{\beta_{\lambda}}{4},
$$

to the lowest order in the $\delta$-expansion the slow roll parameters vanish. As can be seen from (3.6) this corresponds to an extremum of the potential, and $\epsilon=0$ at all orders. For SM Higgs inflation $\lambda_{\max } \sim 5 \times 10^{-5}$. For energies well below the Planck scale the quartic coupling $\lambda(t)$ is a monotonically decreasing function, and there is at most one extremum which is a maximum as ${ }^{8}$

$$
\left.\eta\right|_{\lambda=\lambda_{\max }}=-\frac{8}{3} \frac{\delta^{2}(1+\delta)^{2}\left(1+\frac{\beta_{\lambda}^{\prime}}{4 \beta_{\lambda}}\right)}{\left(1+\frac{1+\delta}{6 \xi}\right)\left(1+\delta+\frac{\beta_{\xi}}{2 \xi}\right)^{2}}<0 .
$$

If the potential develops a maximum in the inflationary regime, 60 efolds of inflation may still occur if the potential near the maximum is flat enough. We will refer to this possibility

\footnotetext{
${ }^{8}$ Close to the Planck scale there is the possibility of a second extremum, a minimum, in the potential. This opens the possibility for inflation near an inflection point. We comment on this in section 3.2.
} 
as "hilltop inflation". To end up in the electroweak vacuum of the Higgs potential, this should happen for field values $\phi<\phi_{\max }$ where $\phi_{\max }$ is the field value at the maximum (this possibility thus constrains the initial field values); this corresponds to the region where $F>0$ is positive. Note that the $\delta$-expansion breaks down close to the maximum, when

$$
F \approx\left(1+\frac{1}{4} \frac{\beta_{\lambda}}{\lambda}\right) \sim \delta\left(1+\frac{\beta_{\lambda}^{\prime}}{4 \beta_{\lambda}}\right)
$$

and the first order term of $\eta$ in (3.7) becomes comparable to the $\delta^{2}$ term in (3.11) (we took the $\xi \gg 1$ limit).

Introduce the notation that the subscript $\star$ denotes the value of the parameters when observable scales leave the horizon, $N_{\star}$ number of efolds before the end of inflation. We distinguish three possibilities.

1. If $F_{\star} \gtrsim \delta_{\star}$ inflation takes place on a flat plateau, and there is no maximum. ${ }^{9}$ This is the case for coupling values $\lambda_{\star} \gtrsim 5 \times 10^{-5}$.

2. If $F_{\star} \lesssim \delta_{\star}$ there is a maximum in the potential. If the maximum is flat enough, hilltop inflation takes place close to the maximum at field values $\phi<\phi_{\max }$. This is the case for coupling values $\lambda_{\star} \sim 5 \times 10^{-5}$.

3. The potential near the maximum is too steep to support $N_{\star}=60$ efolds of inflation.

In this section we will discuss case 1, inflation on the flat plateau. The discussion of case 2 , hilltop inflation, is postponed till the next section. ${ }^{10}$ The value $F_{\star} \sim \delta_{\star}$ divides the two regimes, as follows from (3.12); this is in agreement with our numerical results, which are presented in 3.2. The slow roll parameters (3.7) are affected by the running of the couplings, and corrections may become sizeable for small $\lambda$. However, to calculate the inflationary observables, the slow roll parameters are to be evaluated at the field value $\phi_{\star}$ at which the observable scales leave the horizon. This field value also gets corrected by the running, and as we will show now, these corrections exactly cancel, such that the inflationary predictions are to leading order in the $\delta$-expansion not affected by the running of the couplings.

Let's thus compute the number of efolds $N_{\star}$ before the end of inflation, which is given by

$$
N_{\star} \simeq \int^{h_{\star}} \mathrm{d} h \frac{1}{\sqrt{2 \epsilon}} \simeq \frac{\sqrt{3}}{\left|F_{\star}\right| \sqrt{8\left(1+\frac{1}{6 \xi_{\star}}\right)}} \int^{h_{\star}} \mathrm{d} h \delta^{-1}=\frac{1}{\delta_{\star}\left|F_{\star}\right|} \frac{3}{4} .
$$

On the flat plateau $F>0$ and we can drop the absolute signs. Here we assumed that $F$ and $\xi$ is to first approximation field-independent and we have taken it out of the integral.

\footnotetext{
${ }^{9}$ Note that $\lim _{\phi \rightarrow \infty} \mu^{2}=1 / \xi$ approaches a constant, and the running comes to a halt. For $F_{\star}>\delta_{\star}$ the asymptotic value of $\lim _{\phi \rightarrow \infty} \lambda(t)$ exceeds the critical value (3.10).

${ }^{10}$ It may happen that threshold corrections kill Higgs inflation, in that the corrections to the RGEs bring the model from case 1 to case 3 . The statement we want to make in this paper is that when inflation happens, the predictions are robust and insensitive to UV corrections (except for some possible fine-tuned parameters that allow for hilltop/inflection point inflation).
} 
This gives the leading term in the $\delta$-expansion, as we now quickly explain. The efolds integral can be rewritten as follows

$$
N_{\star}=\frac{3}{2} \int^{\phi^{\star}} \mathrm{d} \phi \frac{\xi}{|F|} \phi+O(\sqrt{\delta})=\frac{3}{2} \int^{\phi_{\star}} \mathrm{d} \phi \phi\left(D_{\star}+\left.\frac{d D}{d \phi}\right|_{\phi=\phi_{\star}}\left(\phi-\phi_{\star}\right)+. .\right) .
$$

In the first step we used the field space metric to express the integral in terms of the (noncanonical) field $\phi(2.5)$, in the second step we defined $D\left(\beta_{i}, \lambda, \xi\right) \equiv \xi|F|^{-1}$ and expanded the integrand around $\phi_{\star}$. The first term in the expansion is the only one considered in (3.13). It gives a contribution of the form

$$
\int^{\star} \mathrm{d} \phi \phi D_{\star} \propto \phi_{\star}^{2} \propto O\left(\delta_{\star}^{-1}\right)
$$

The second term in the expansion takes the form $\left(f_{i} \equiv\left\{\lambda, \xi, \beta_{\lambda}, \beta_{\xi}\right\}\right)$,

$$
\left.\int^{\phi_{\star}} \mathrm{d} \phi \frac{d D}{d \phi}\right|_{\star} \phi\left(\phi-\phi_{\star}\right)=\left.\int^{\star} \mathrm{d} \phi \phi\left(\phi-\phi_{\star}\right)\left(\frac{d D}{d f_{i}} \frac{d f_{i}}{d t} \frac{d t}{d \phi}\right)\right|_{\star} \propto \phi_{\star}^{3} \delta_{\star}^{\frac{3}{2}} \propto O\left(\delta_{\star}^{0}\right),
$$

where we used $d t /\left.d \phi\right|_{\star} \propto \delta_{\star}^{\frac{3}{2}}$ from (3.5). It is higher order in the $\delta$ expansion and can be neglected. We also neglected the lower bound of the integral; this correction is likewise higher order in $1 / N_{\star} \sim \delta_{\star}$. Using (3.13) from the COBE normalization we get [5]

$$
\left(\frac{V}{\epsilon}\right)_{\star}=\frac{4}{3} \frac{\lambda}{\xi^{2} N_{\star}^{2}}=(0.027)^{4} \quad \Rightarrow \quad \frac{\xi\left(t_{\star}\right)}{\sqrt{\lambda\left(t_{\star}\right)}}=5 \times 10^{4} .
$$

Plugging (3.13) in the expressions (3.7) gives the spectral index and tensor-to-scalar ratio

$$
n_{s}=1+2 \eta+\mathcal{O}\left(\delta^{2}\right)=1-\frac{2}{N_{\star}}+\mathcal{O}\left(\delta^{2}\right)
$$

and

$$
r=16 \epsilon=\frac{12}{N_{\star}^{2}}\left(1+\frac{1}{6 \xi}\right)+\mathcal{O}\left(\delta^{3}\right)
$$

The COBE normalization can always be fit by choosing the non-minimal coupling appropriately. All parameters in the model are then fixed. For the large non-minimal couplings needed $\left(\xi_{\star}>10^{2}\right)$, the spectral index and tensor-to-scalar ratio only depend on the number of efolds. All dependence on the beta-function has cancelled in the final expression, and the results are identical to those for classical Higgs inflation. This means that the results for plateau inflation are very robust: they are independent from the running, and thus insensitive to UV physics that change the running, and also independent of the electroweak boundary conditions on the couplings.

At next order in the $\delta$ expansion the beta-function do enter, see appendix $\mathrm{B}$, but this is too small an effect to be measured. 


\subsection{Inflation near the maximum}

As discussed previously, for $F_{\star}>\delta_{\star}$ inflation takes place on the flat part of the potential and the inflationary observables are insensitive to the running of the couplings, to first order they depend only on the number of efolds. Here we discuss the case $F_{\star}<\delta_{\star}$. When $F=0$ the potential develops a maximum. Requiring the Higgs field to end up in the electroweak vacuum, inflation should take place at field values before the maximum, where $F>0$. We expect Hilltop inflation to be highly sensitive to the form of the potential and thus to the details of EW boundary conditions and to threshold corrections. Unfortunately, because of this sensitivity, it is hard to obtain analytical expressions, and we will only present numerical results. We point out that even though hilltop inflation is sensitive to the UV completion, it only occurs for very fine-tuned boundary conditions. Thus the numerical results presented in this section confirm our statement that the predictions for Higgs inflation are remarkably robust, and they verify the analytical result of section 3.1. For more details on our numerical implementation see section 2 and in particular 2.4.

Let's start by considering just the SM running, and turn off all threshold corrections. We can tune $F$ small by adjusting the boundary conditions at the electroweak scale. We choose to decrease the Higgs mass, while keeping the top mass and gauge couplings fixed. ${ }^{11}$ Our results are summarized in table 1. They agree with the discussion above. For large enough Higgs mass values, inflation takes place on the flat plateau, and $n_{s}$ and $r$ are independent on the running. In some fine-tuned range of Higgs mass values, inflation can happen near a maximum; in this case the inflationary results depend sensitively on the EW boundary conditions. For an even smaller Higgs mass the maximum is too steep and 60 efolds of inflation is not possible. In figure 1 we show an example potential for inflation on the plateau and for inflation near the maximum, ${ }^{12}$ the parameters corresponding to the first and last line of table 1 . Our numerical results agree with similar studies in the literature $[32,37]$.

Now turn on the threshold corrections. We choose $m_{t}=171 \mathrm{GeV}, m_{h}=125.5 \mathrm{GeV}$ and did 500 simulations with Wilson coefficients randomly chosen between $c_{i}=\operatorname{Random}[-10,10]$. We found 382 times that inflation takes place on the flat plateau, and the other 118 times there was no inflationary solution. Hilltop inflation does not happen. The spread in spectral index, tensor-to-scalar ratio, $\xi_{0}$ and kick $\Delta \lambda$ for the successful models are shown in figure 2. The kick in $\lambda$ is defined with respect to the reference set-up without threshold corrections, corresponding to the highlighted line in table 1. Define $\left(\lambda_{\star}^{\mathrm{SM}}, t_{\star}^{\mathrm{SM}}, \xi_{0}^{\mathrm{SM}}\right)=$ $\left(9.0 \times 10^{-4}, 33.55,1417\right)$ for this model, with $t=\ln \left(\mu / m_{t}\right)$ and $\xi_{0}$ the boundary condition $\xi(1 / \xi)=\xi_{0}$. We then define the kick in $\lambda$ for the models with threshold corrections as

$$
\Delta \lambda=\lambda\left(t_{\star}^{\mathrm{SM}}\right)-\lambda_{\star}^{\mathrm{SM}}, \quad \text { for } \xi_{0}=\xi_{0}^{\mathrm{SM}} .
$$

For our run of 500 simulations, the average kick is upwards $\langle\Delta \lambda\rangle=1.5 \times 10^{-3}$ with standard deviation $\sigma=3.9 \times 10^{-3}$; the average absolute kick size is $\langle|\Delta \lambda|\rangle=2.3 \times 10^{-3}$. If the kick

\footnotetext{
${ }^{11}$ We choose $m_{t}=171 \mathrm{GeV}$ which is about $2 \sigma$ below its central value, to avoid that the quartic coupling becomes negative before inflation.

${ }^{12}$ For these plots we numerically inverted $t(\phi)$. This inversion is not needed to calculate $n_{s}$ and $r$, which is done with $t$ as the clock variable.
} 

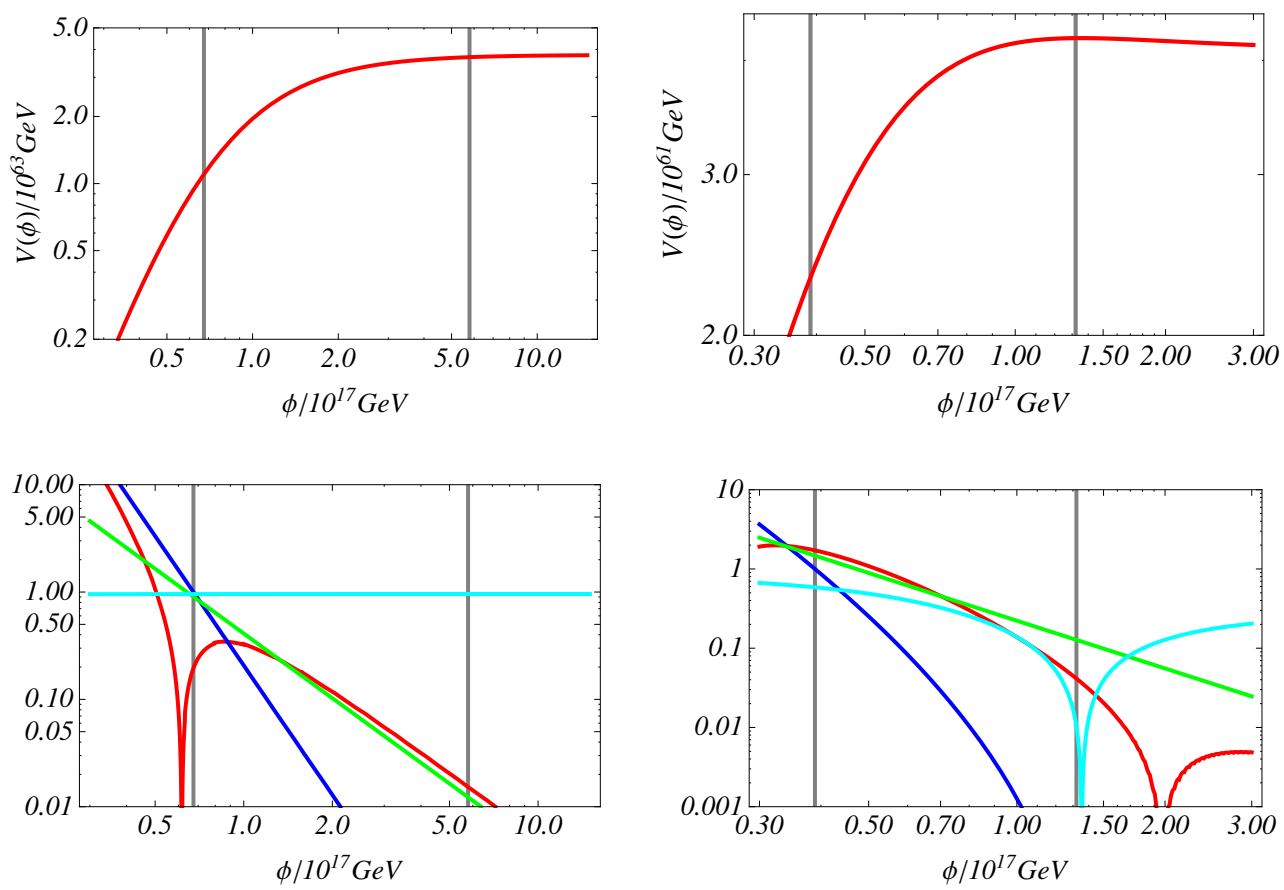

Figure 1. Top left the potential $V(\phi)$ for inflation with $m_{t}=171 \mathrm{GeV}$ and $m_{h}=125.5 \mathrm{GeV}$; the grey vertical lines correspond to $\phi_{\star}$ and $\phi_{\text {end }}$ respectively (i.e. the beginning and end of inflation). Bottom left shows $|\eta|$ (red), $\epsilon$ (blue), $\delta$ (green) and $F$ (cyan) respectively for the same top mass. Right top and bottom, same plots but for $m_{t}=171 \mathrm{GeV}$ and $m_{h}=125.245 \mathrm{GeV}$.

\begin{tabular}{|l|c|c|c|c|c|c|c|}
\hline$m_{h}(\mathrm{GeV})$ & $h_{\star}$ & $\lambda_{\star}$ & $\xi_{\star}$ & $F_{\star}$ & $\delta_{\star}$ & $n_{s}$ & $r$ \\
\hline 127 & 0.15 & $6.3 \times 10^{-3}$ & 3863 & 0.99 & 0.01 & 0.968 & $3.0 \times 10^{-3}$ \\
\hline 126 & 0.18 & $2.7 \times 10^{-3}$ & 2505 & 0.98 & 0.01 & 0.968 & $3.0 \times 10^{-3}$ \\
\hline 125.5 & 0.24 & $9.0 \times 10^{-4}$ & 1451 & 0.96 & 0.01 & 0.968 & $3.0 \times 10^{-3}$ \\
\hline 125.3 & 0.33 & $1.9 \times 10^{-4}$ & 667 & 0.84 & 0.01 & 0.968 & $2.9 \times 10^{-3}$ \\
\hline 125.26 & 0.34 & $4.2 \times 10^{-5}$ & 344 & 0.42 & 0.03 & 0.970 & $2.4 \times 10^{-3}$ \\
\hline 125.255 & 0.20 & $3.1 \times 10^{-5}$ & 451 & 0.12 & 0.06 & 0.968 & $9.5 \times 10^{-4}$ \\
\hline \hline 125.253 & 0.13 & $3.3 \times 10^{-5}$ & 730 & 0.05 & 0.08 & 0.958 & $3.7 \times 10^{-4}$ \\
\hline 125.25 & 0.09 & $3.7 \times 10^{-5}$ & 1314 & 0.07 & 0.12 & 0.941 & $1.2 \times 10^{-4}$ \\
\hline 125.245 & 0.05 & $4.3 \times 10^{-5}$ & 2678 & 0.01 & 0.12 & 0.917 & $3.4 \times 10^{-5}$ \\
\hline
\end{tabular}

Table 1. Inflationary parameters for different Higgs mass while $m_{t}=171 \mathrm{GeV}$ is kept fixed, in the absence of threshold corrections. Above the double line the potential has a flat plateau, below the line the potential develops a maximum. For $m_{h}<125.245$ no inflationary solutions with $N_{\star}=60$ efolds exists.

is upwards, or downwards but not so large, plateau inflation is still possible. The value $\xi_{0}$, which is a free parameter, has to be adjusted with respect to the reference model, to fit the power spectrum (3.17). However for large kicks downwards this is no longer possible, and the potential is too steep for all $\xi$-values. The critical kick dividing the successful models 

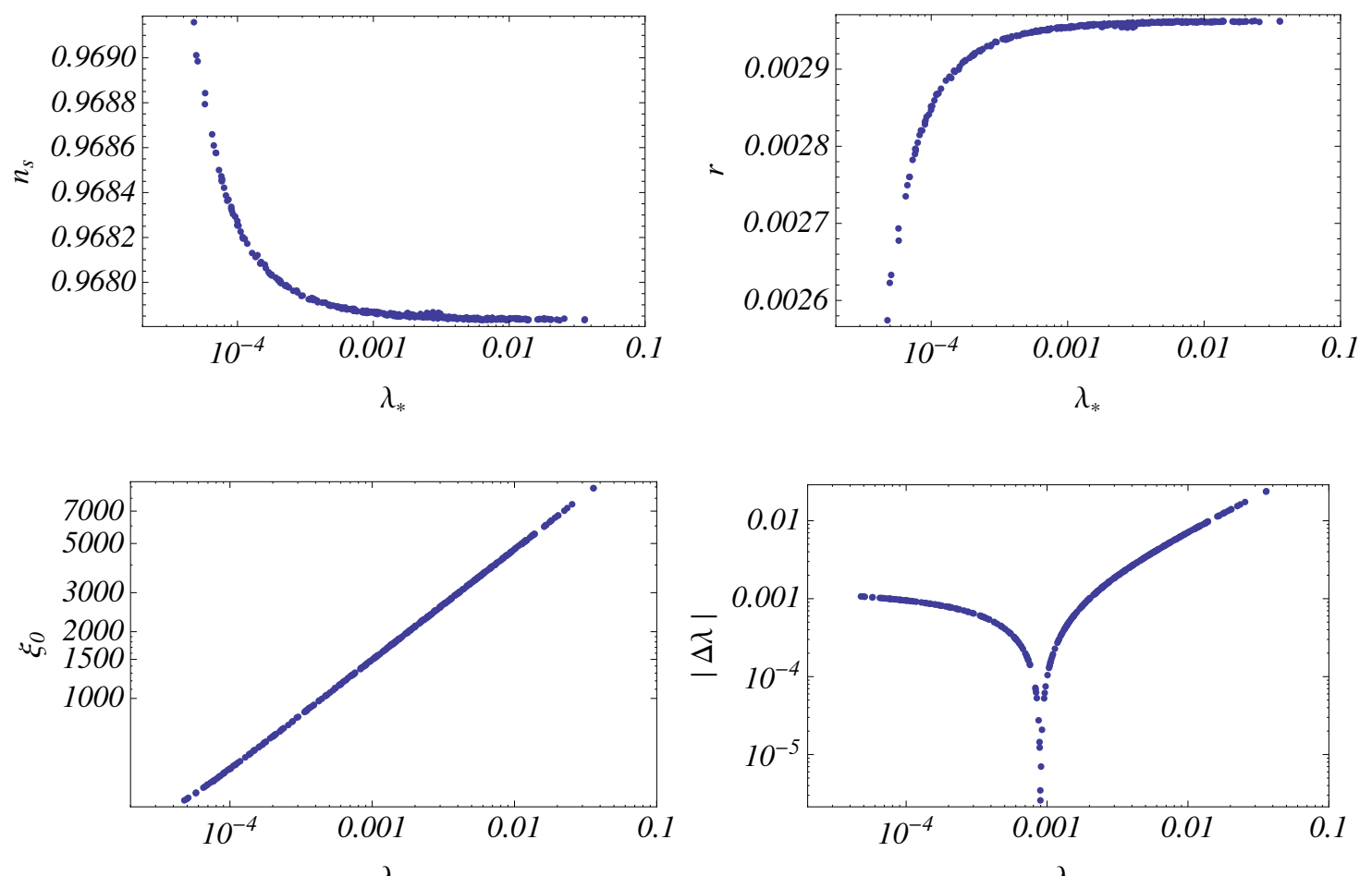

$\lambda_{*}$

$\lambda_{*}$

Figure 2. The spread in spectral index $n_{s}$, tensor-to-scalar ratio $r, \xi_{0}$ and $\Delta \lambda$ as a function of $\lambda_{\star}$ for the 382 succesfull models with threshold corrections $c_{i}=$ Random[-10,10].

from the unsuccessful ones is

$$
\Delta \lambda^{\text {crit }}=-1.1 \times 10^{-3} \Rightarrow \lambda_{\star}^{\mathrm{SM}}+\Delta \lambda^{\text {crit }}=-1.7 \times 10^{-4}
$$

As mentioned, we do not find any examples of hilltop inflation in our 500 simulations with threshold corrections, in contrast to the pure SM running. One can either generate a kick, with respect to the reference model, by changing the boundary conditions at the electroweak scale (e.g. changing the EW top/Higgs mass) or by turning on threshold corrections. Adding thus a kick to the reference model, the CMB power spectrum constraint is no longer satisfied; we retune $\xi_{0}$ to fit the CMB data. Figure 3 shows the result, it plots $\xi_{0}$ for downwards kicks $\Delta \lambda<0$. The reference model is again $m_{t}=171 \mathrm{GeV}, m_{h}=125.5 \mathrm{GeV}$, that is the highlighted line in table 1 . In this plot the green line correspond to pure SM running and different values of the EW top mass, the red line for SM running and different values of the EW Higgs mass (corresponding to the results in table 1), and the blue line for fixed top and Higgs mass but a kick generated by threshold corrections. Inflation near the maximum only happens in the first two cases for the small kick interval where $\xi_{0}$ increases again (i.e. where the red and blue line increase).

It matters whether the kick is produced by EW boundary conditions or by threshold corrections. In the 2 nd case, inflation is possible for larger kick values. This can be understood as follows. For SM running without threshold corrections, changing $\xi_{0}$ mainly affects the size of the power spectrum, but it has only a small effect on the running. In 


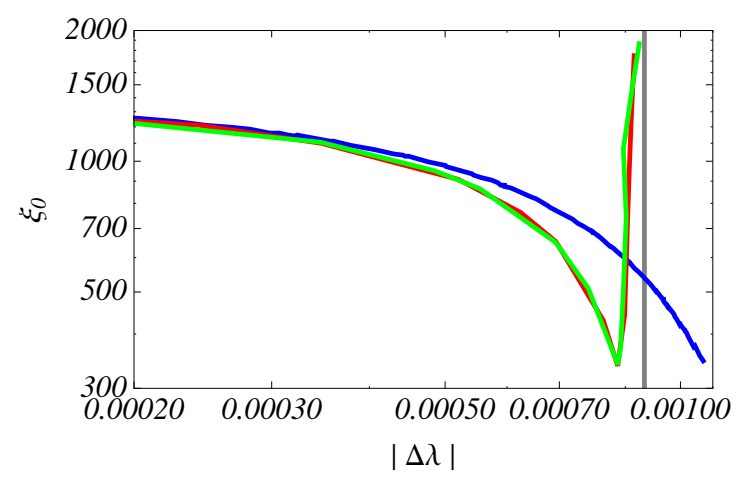

Figure 3. The boundary value $\xi_{0}$ for the non-minimal coupling vs. the kick $|\Delta \lambda|$ for SM running (green/red) and SM running with threshold corrections (blue). In the former case the kick is from changing the top/Higgs mass at the electroweak scale, whereas in the latter it is due to the threshold corrections. The kick is downwards $\Delta \lambda<0$. The vertical grey line corresponds to the value $\lambda_{*}^{\mathrm{SM}}+\Delta \lambda=0$.

contrast, for the set-up with threshold corrections, changing $\xi_{0}$ will both affect the power spectrum and the running. Indeed, $\mu \sim 1 / \xi_{0}$ is the scale where the kick is produced. For a smaller $\xi_{0}$ this happens at a higher scale, where the value of $\lambda(\mu)$ is smaller and since the size of the kick is proportional to $\lambda(\mu)$, this results in a smaller kick. Hilltop inflation is only possible if the curvature near the maximum is tuned small. This depends on the details of the potential. It is no surprise that this gives slightly different results for SM running, and SM including threshold corrections, even for a similarly sized kick.

No matter what the exact form of the threshold corrections is, if the kick is not too large $\left(\Delta \lambda+\lambda_{*}^{\mathrm{SM}}>0\right)$ inflation takes place on the flat plateau with universal predictions for the observables. The larger $\lambda_{*}^{\mathrm{SM}}$ is without threshold corrections, the larger kick is needed to disrupt inflation, which is only possible for large Wilson coefficients of the nonrenormalizable operators. Consider for example the first line in table 1 , with $\lambda_{*}^{\mathrm{SM}}=$ $6.3 \times 10^{-3}$. Also for this case we did 500 simulations with random Wilson coefficients, choosing $c^{\max }=20$ with $c_{i}=\operatorname{random}\left[-c^{\max }, c^{\max }\right]$. We found that 61 out of 500 times the downwards kick was large, and inflation no longer possible; we found no examples of hilltop inflation.

Choosing natural values for the Wilson coefficients $c^{\max } \sim 1$ the effect of the threshold corrections on the running is small. Nevertheless, there might be additional sources of threshold corrections. If they only affect the potential via modifications of the running, our results apply: (except from some possible fine-tuned cases near a maximum) inflation takes place on the plateau and the observables have universal values. Our choice of higher order terms in the Lagrangian (2.8) with $c^{\max } \sim 10$ can be viewed as a (specific) parameterization of the kick in $\lambda$ due to all possible threshold corrections.

Finally we would like to comment on the possibility of Higgs inflation near an inflection point, as has been discussed in the literature $[57,58]$. Close to the Planck scale the potential may develop a second minimum. For fine-tuned boundary conditions the maximum and minimum merge into an inflection point with $V_{h}=V_{h h}=0$, where inflation can take place. 
Such solutions only exist for relatively small non-minimal coupling $\xi=\mathcal{O}(10)$; this is because the renormalization scale is bounded $\mu<1 / \sqrt{\xi}$, and only for small $\xi$ large enough scales can be reached where the Landau pole becomes important. Because of the large scales involved, inflection point inflation can give rise to a large gravitational wave signal (these models were motivated by the BICEP results). In our numerics we did not search for this possibility, and it is not included in our results.

\section{Conclusions}

In Higgs inflation the unitarity cutoff, signaling the breakdown of the effective theory, is well below the Planck scale and introducing an UV completion is demanded by the consistency of the theory. This raises the question how sensitive the CMB predictions are to the UV completion above the cutoff scale. In this paper we have shown that as long as the UV corrections do not affect the inflaton potential at tree level but only enter at loop level via corrections to the renormalization group equations, the inflationary predictions are (almost) unaffected. Indeed, as we proved analytically in section 3.1, to leading order in the slow roll expansion all dependence on the running cancels, and thus the predictions are insensitive to threshold corrections. The spectral index and tensor-to-scalar ratio are exactly the same as for the classical, tree level potential, which is in excellent agreement with data.

The inflationary predictions are universal if inflation takes place on the flat plateau of the potential. However, it may happen that due to the running of the couplings the potential develops a maximum. Inflation near the maximum will depend on the details of the RGE evolution and thus on the UV completion. The perturbative expansion used in section 3.1 does not capture this case, and we used a numerical analysis to also study the possibility of hilltop inflation, where we parameterized the threshold corrections by a specific set of higher order operators in the Lagrangian (2.8). Our numerical analysis confirms our analytical results for inflation on the flat plateau of the potential. We further found that hilltop inflation is a possibility, but it only happens for very fine-tuned boundary conditions (the top/Higgs mass at the electroweak scale, and the Wilson coefficients of the non-renormalizable operators). Indeed, for our run of 500 simulations with randomly chosen Wilson coefficients (taken large enough, such that the effect on the running is appreciable - see section 3.2 for more details), we found 382 times plateau inflation, and 118 times inflation was spoiled as the potential became unstable at low field values and the corresponding maximum was too steep to support 60 efolds of inflation. In this run, we did not find a single instance of hilltop inflation. We conclude that, apart from the very fine-tuned case of inflation near the maximum, if inflation happens, the predictions are the same as those derived from the classical potential (1.1).

A previous study of threshold correction to HI has been done in [22]. They concluded that Higgs inflation is extremely sensitive to the UV completion, which was modelled by the same set of higher order operators (2.8) [42]. We expect that the difference is mainly due to the choice of the renormalization prescription. While [22] allows for both prescription 1 and 2 in their numerical analysis, we showed analytically (and confirmed 
numerically) that for our choice of $\mu$, which has been discussed extensively in $(2.3),{ }^{13}$ such dependence does not arise in general. Furthermore, there are some slight differences in the numerical implementation, for example the set of RGEs for the inflationary regime, and the parameterization of the unitarity cutoff. However, the main conclusion that inflation on the flat plateau of the potential is insensitive to UV physics, does not depend on these choices. As mentioned above, we do find deviations from the universal predictions if inflation occurs near a maximum of the potential. We studied numerically the fine-tuned parameter space for hilltop inflation, which depends sensitively on the boundary conditions as well as on the UV completion - and thus also on the specifics of the numerical implementation. For standard model inflation, without threshold corrections, our numerical results agree with earlier work $[32,37]$.

We conclude with a small remark. It is well known that for the central values of the electroweak scale top and Higgs mass the Higgs potential becomes unstable at $\phi \sim$ $10^{10} \mathrm{GeV}[6-12]$, well before the potential flattens in Higgs inflation. The top/Higgs mass values separating a stable from an unstable Standard Model Higgs potential are close to those separating Higgs inflation from models where inflation is not possible. There are small differences with respect to SM running (without a non-minimal coupling), because 1) we include threshold effects, 2) we run until the inflationary scale and not the Planck scale, and 3) the RGE equations get modified in the mid and large field regime. As expected the measured Higgs and top masses [59] are surprisingly close to the border separating the regions where the Higgs boson can or cannot be the inflaton.

\section{Acknowledgments}

We thank Sander Mooij, Enrico Morgante, Subodh Patil, Mike Trott and Cliff Burgess for very helpful discussions. The authors are funded by the Netherlands Foundation for Fundamental Research of Matter (FOM) and the Netherlands Organisation for Scientific Research (NWO).

\section{A Effective action and renormalization group improvement}

In Higgs inflation the canonically normalized field $h$ and the field $\phi$ appearing in the Einstein frame action (2.4) are related by a non-trivial field space metric (2.5). Although it is possible to write $h(\phi)$ in closed form [21,60], this relation can only be inverted in certain limits. Therefore, the potential in terms of the canonical field $h$ can not be expressed in an analytical form over the whole field domain, and it is often more convenient to work with the $\phi$-field (as we did, for example, in section 3).

The RG improved potential is usually defined in terms of the canonical field. In this appendix we will show that the usual procedure of substituting each coupling with its running counterpart can also be used for the non-canonical $\phi$ field, and the RG improved

\footnotetext{
${ }^{13} \mathrm{As}$ argued in (2.3) and in (A), prescription 1 is the only consistent renormalization scale parametrization to study the RG improved potential in the Einstein frame.
} 
potential is obtained as ${ }^{14}$

$$
V_{E}=\frac{\lambda \phi^{4}}{\left(1+\xi \phi^{2}\right)^{2}} \longrightarrow \frac{\lambda(t) \phi^{4}}{\left(1+\xi(t) \phi^{2}\right)^{2}}
$$

We first quickly review how the RG improved potential can be defined for canonical fields, and then generalize to the case with non-trivial field space metric.

\section{A.1 Canonical kinetic sector}

Consider first the SM without the non-minimal coupling, the Higgs kinetic term is canonical.

The effective action $\Gamma\left[\phi_{\mathrm{cl}}\right]=W[J]-\int J \phi_{\mathrm{cl}}$, with $\phi_{\mathrm{cl}}=\delta W / \delta J$ describes the quantum corrected dynamics of the background field, since

$$
\left.\frac{\delta \Gamma\left[\phi_{\mathrm{cl}}\right]}{\delta \phi_{\mathrm{cl}}}\right|_{\bar{\phi}_{\mathrm{cl}}}=0, \quad \bar{\phi}_{\mathrm{cl}}=\langle\Omega|\phi| \Omega\rangle \equiv\langle\phi\rangle,
$$

i.e. the vacuum expectation value is given by minimizing the effective action. $\Gamma\left[\phi_{\mathrm{cl}}\right]$ has the following form

$$
\Gamma\left[\phi_{\mathrm{cl}}\right]=S_{r}\left[\phi_{\mathrm{cl}}\right]+\Delta S_{c}\left[\phi_{\mathrm{cl}}\right]+\Gamma^{1-\mathrm{loop}}+\Gamma^{2-\text { loop }}+\ldots
$$

The first term is the classical renormalized action, $\Delta S_{c}$ contains the counterterms and the third term represent the one loop correction, ${ }^{15}$

$$
\Gamma^{1-\text { loop }}=\frac{i}{2} \int d^{4} x \sum_{i}(-1)^{F_{i}} S_{i} \operatorname{Tr} \ln \left(D_{i}+m_{i}^{2}\left(\phi_{\mathrm{cl}}\right)\right)
$$

$S_{i}$ counts the degrees of freedom of each particle with mass $m_{i}, F_{i}$ is 1 for fermions and 0 for bosons. $\Gamma$ is finite (physical amplitudes are derived from it), as the infinities from the loop contributions are eliminated by the counterterms.

Usually one is interested in finding the space-time independent vacuum state. Thus $\phi_{\mathrm{cl}}$ is taken constant, and the one loop contribution to $\Gamma$ can easily be computed since the operators inside the log become diagonal in momentum representation. The effective action for a constant background field reduces then to the effective potential given by the tree level contribution plus the well known Coleman-Weinberg corrections [44]

$$
\begin{aligned}
\Gamma\left[\phi_{\mathrm{cl}}\right] & =-\int d^{4} x V_{\mathrm{eff}}\left[\phi_{\mathrm{cl}}\right] \\
& =-\int d^{4} x\left[V_{\mathrm{tree}}\left(\phi_{\mathrm{cl}}\right)+\frac{1}{64 \pi^{2}} \sum_{i}(-1)^{F_{i}} S_{i} m_{i}^{4}\left(\phi_{\mathrm{cl}}\right)\left[\ln \left(\frac{m_{i}^{2}(\phi)}{\mu^{2}}\right)-c_{i}\right]\right]+. .
\end{aligned}
$$

\footnotetext{
${ }^{14}$ That this is possible is not immediately obvious. In the large field regime $\phi=\frac{1}{\sqrt{\xi}} \mathrm{e}^{h / \sqrt{6}}$; as this relation depends explicitly on a coupling, the potential in terms of the canonical field has a different coupling dependence (it only depends on the combination $\lambda / \xi^{2}$ ), and at first sight it might seem that the prescription of making all couplings running (A.1) differs when done in terms of $h$ or $\phi$. We also did the calculation of the inflationary predictions in terms of the canonical field, and at leading order in the $\delta$-expansion found identical results to those presented in section 3.

${ }^{15}$ We only consider one-loop corrections in the inflationary regime.
} 
in the $\overline{\mathrm{MS}}$ renormalization scheme. Here $V_{\text {tree }}$ is the potential at tree level, $\mu$ is the normalization scale, and $c_{i}=3 / 2$ for fermions and scalars and $c_{i}=5 / 6$ for gauge bosons. Minimizing the effective potential gives the vev for a constant background scalar field.

The perturbative expansion breaks down when the logs in the CW potential become large. This problem can be avoided by rewriting the effective action as a formal solution of the RG equation. In fact, due to the invariance of the theory with respect to the renormalization procedure $\Gamma$ satisfies the Callan-Symanzik equation [61, 62]

$$
\left(\mu \frac{\partial}{\partial \mu}+\beta_{i} \frac{\partial}{\partial g_{i}}-\gamma \int d^{4} x \phi_{\mathrm{cl}} \frac{\delta}{\delta \phi_{\mathrm{cl}}}\right) \Gamma\left[\phi_{\mathrm{cl}}, g_{i}, \mu\right]=0 .
$$

The formal solution is given by straightforwardly applying the method of characteristics [63]

$$
V_{\mathrm{eff}}\left(\phi_{\mathrm{cl}}, g_{i}, \mu\right)=V_{\mathrm{eff}}\left(\phi(t), g_{i}(t), \mu(t)\right) \equiv V(t),
$$

with

$$
\phi(t)=\rho(t) \phi_{\mathrm{cl}}, \quad \frac{d g_{i}(t)}{d t}=\beta_{i}\left(g_{j}(t)\right), \quad \mu(t)=\mu e^{t}, \quad \frac{d \ln \rho(t)}{d t}=-\gamma\left(g_{j}(t)\right) .
$$

Here $g_{i}$ represent the generic couplings and $\gamma$ is the anomalous dimension of the Higgs field. We also assume the initial conditions $\rho(0)=1, g_{i}(t)=g_{i}, \mu(0)=\mu$.

The power of the RG is the fact that we can choose the functional form of $t=t(\phi)$ in such a way that the perturbation series for $V(t)$ converges more rapidly than the one for $V(0)$. This can be made explicit by choosing $\mu(t)$ such that the logs in the CW potential are minimized, i.e. $\mu(t)^{2} \sim m_{i}^{2}\left(\phi_{\mathrm{cl}}\right)$.

During inflation the background field is rolling down its potential, i.e. $\phi_{\mathrm{cl}}(t)$ is not constant. As a result the masses appearing in expressions like (A.4) are not constant and the 1-loop contributions must be calculated in spacetime-dependent perturbation theory [64-66]. The effective action assumes the generic form

$$
\Gamma\left[\phi_{\mathrm{cl}}\right]=-\int d^{4} x\left[\frac{1}{2} Z\left(\phi_{\mathrm{cl}}\right) \partial_{\mu} \phi_{\mathrm{cl}} \partial^{\mu} \phi_{\mathrm{cl}}+V_{\mathrm{eff}}\left[\phi_{\mathrm{cl}}\right]+\ldots\right]
$$

where the dots are for higher derivatives terms $\sim Y\left(\phi_{\mathrm{cl}}\right)\left(\partial \phi_{\mathrm{cl}}\right)^{4}+$. that we can safely neglect in the slow roll approximation, and $Z=Z\left(\phi_{\mathrm{cl}}, g_{i}, \mu\right)$. Applying the Callan-Symanzik equation to the kinetic term gives, after some integrations by part, the following expression

$$
\left(\mu \frac{\partial}{\partial \mu}+\beta \frac{\partial}{\partial \lambda}-\gamma\left(2+\phi_{\mathrm{cl}} \frac{\partial}{\partial \phi_{\mathrm{cl}}}\right)\right) Z\left(\phi_{\mathrm{cl}}, g_{i}, \mu\right)=0 .
$$

The formal solution can be written as

$$
Z\left(\phi_{\mathrm{cl}}, g_{i}, \mu\right)=Z\left(\phi(t), g_{i}(t), \mu(t)\right) \rho^{2}(t) \equiv Z_{\mathrm{eff}}(t) .
$$

where $\left\{\phi(t), \rho(t), g_{i}(t), \mu(t)\right\}$ are given in (A.8), with the same initial conditions. In the leading order approximation ${ }^{16}$

$$
Z_{\mathrm{eff}}(t) \approx \rho^{2}(t)=e^{-2 \int_{0}^{t} \gamma\left(t^{\prime}\right) d t^{\prime}}
$$

\footnotetext{
${ }^{16}$ Since also $Z$ depends on a series of logarithms, the best choice of $t$ to minimize them in the effective potential is to set $Z$ to one at the leading order.
} 
and the improved effective action becomes

$$
\Gamma=-\frac{1}{2} \int\left[Z_{\mathrm{eff}}(t)\left(\partial_{\mu} \phi_{\mathrm{cl}}\right)^{2}+V(t)\right]
$$

As usual, it is convenient to use a canonical field redefinition, i.e. $Z_{\mathrm{eff}}^{1 / 2} d \phi_{\mathrm{cl}}=d \phi_{\mathrm{can}}$. This is useful for two reasons: the equations become simpler and the gauge dependence in the potential is significantly reduced (see the discussion in ref. [67]). Consider for example the tree level potential $V_{\text {tree }}=\lambda \phi_{\mathrm{cl}}^{4} / 4$. The improved effective action is

$$
\Gamma=-\int\left[\frac{Z_{\mathrm{eff}}(t)}{2}\left(\partial_{\mu} \phi_{\mathrm{cl}}\right)^{2}+\frac{\lambda(t)}{4} \rho^{4}(t) \phi_{\mathrm{cl}}^{4}+. .\right]=-\int\left[\frac{1}{2}\left(\partial_{\mu} \phi_{\mathrm{can}}\right)^{2}+\frac{\lambda(t)}{4} \phi_{\mathrm{can}}^{4}+. .\right]
$$

where in the last step we used (A.12). This explains why, with proper choice of the function $t$ (or equivalently $\mu(t)$ ), the effect of the renormalization group can be summarized, from an operative point of view, in taking the action and making the couplings running. In this example $\lambda(t)=d \beta_{\lambda}(\lambda(t)) / d t$.

\section{A.2 Non canonical kinetic sector}

Let us now see how the previous discussion can be generalized to the non-minimal kinetic terms in Higgs inflation. The Einstein frame effective action is

$$
\Gamma\left[\phi_{\mathrm{cl}}\right]=-\int d^{4} x\left[\frac{1}{2} Z\left(\phi_{\mathrm{cl}}\right) \gamma_{\phi \phi}\left(\phi_{\mathrm{cl}}, \xi\right) \partial_{\mu} \phi_{\mathrm{cl}} \partial^{\mu} \phi_{\mathrm{cl}}+V_{\mathrm{eff}}\left[\phi_{\mathrm{cl}}\right]+\text { h.o. }\right]
$$

The fermion, gauge boson, Higgs and Goldstone boson masses are given in (2.10), and the field space metric $\gamma_{\phi \phi}$ in (2.5). Even though the kinetic terms are non-canonical, one can define the improved effective action as a formal solution of the RG equations. The result now is that $Z^{\prime} \equiv Z\left(\phi_{\mathrm{cl}}\right) \gamma_{\phi \phi}\left(\phi_{\mathrm{cl}}, \xi\right)$ satisfies an equation of the form (A.10) and its solution can be rewritten, like in (A.11), as

$$
Z_{\text {eff }}^{\prime}(t)=Z\left(\phi(t), g_{i}(t), \mu(t)\right) \gamma_{\phi \phi}(\phi(t), \xi(t)) \rho^{2}(t) \approx \gamma_{\phi \phi}(t) \rho^{2}(t)
$$

The improved effective action takes the form

$$
\Gamma=-\int d^{4} x\left[\frac{1}{2} \rho^{2}(t) \gamma_{\phi \phi}(\phi(t), \xi(t))\left(\partial_{\mu} \phi_{\mathrm{cl}}\right)^{2}+V_{\mathrm{eff}}\left(\phi(t), g_{i}(t), \mu(t)\right],\right.
$$

with $g_{i}$ labeling all SM model couplings plus the non-minimal coupling $\xi . V(t)$ is the effective potential for Higgs inflation rewritten as a solution of the RG equation, i.e (A.7). Now proceed exactly as before in order to rewrite the improved action in terms of a canonical field. Let us do that in two steps. First use the following field redefinition

$$
d \tilde{\phi}_{\mathrm{can}}=\rho(t) d \phi_{\mathrm{cl}} \text {. }
$$

Then for $\phi(t)$ we obtain

$$
\phi(t)=\rho(t) \phi_{\mathrm{cl}}=\exp \left(-\int_{0}^{t} \gamma(t) d t\right) \rho^{-1}(t) \tilde{\phi}_{\mathrm{can}} \approx \tilde{\phi}_{\mathrm{can}},
$$


and the effective lagrangian becomes $\left(\Gamma \equiv \int d^{4} x \mathcal{L}_{\text {eff }}\right)$

$$
\mathcal{L}_{\text {eff }}=-\frac{1}{2} \gamma_{\phi \phi}\left(\tilde{\phi}_{\text {can }}, g_{i}(t), \mu(t)\right)\left(\partial_{\mu} \tilde{\phi}_{\text {can }}\right)^{2}-V_{\text {eff }}\left(\tilde{\phi}_{\text {can }}, g_{i}(t), \mu(t)\right) .
$$

Then define the canonical field via

$$
d \phi_{\mathrm{can}}=\sqrt{\gamma_{\phi \phi}} d \tilde{\phi}_{\mathrm{can}} .
$$

To connect to the notation of the rest of this paper set $\tilde{\phi}_{\text {can }} \equiv \phi$ and $\phi_{\text {can }}=h$. The improved potential in (A.20) is the Higgs inflation quantum improved potential (eq. (A.7)) after the field redefinition (A.18), i.e.

$$
V_{\text {eff }}=\frac{\lambda(t) \phi^{4}}{(1+\xi(t) \phi)^{2}}+\frac{1}{64 \pi^{2}} \sum_{i} S_{i} m_{i}^{4}\left(\phi, g_{i}(t)\right)\left[\ln \left(\frac{m_{i}^{2}\left(\phi, g_{i}(t)\right)}{\mu(t)}\right)-c_{\mathrm{i}}\right] \text {. }
$$

The optimal choice for the renormalization scale, which kills the (dominant) logs of the top and gauge boson loop contributions in the $\mathrm{CW}$ potential, is given by

$$
\mu(t)=m_{t}(\mu \sim E W) \mathrm{e}^{t} \sim \frac{\phi}{\Omega(t)}=\frac{\phi}{\sqrt{1+\xi(t) \phi^{2}}}
$$

where $m_{t}(\mu \sim E W)$ is the top mass measured at the Electroweak scale. This is (2.23), which is our choice for the renormalization scale. It follows that the RG improved potential becomes

$$
V_{\mathrm{eff}}(\phi) \simeq \frac{\lambda(t(\phi)) \phi^{4}}{\left(1+\xi(t(\phi)) \phi^{2}\right)^{2}},
$$

where $\phi=\phi(h)$ through (A.21).

\section{B CMB parameters at higher order in $\delta$}

In this appendix we compute the perturbation spectrum at second order in the slow roll expansion. At this order the results do depend on the running. We check that there is no accidental cancellations or terms blowing up, and that the leading order results are indeed the dominant terms.

In order to compute the CMB parameters $\left(n_{s}, r\right)$ at second order in $\delta=1 /\left(\xi \phi^{2}\right)$ we need the slow roll parameter $\eta$ at 2nd order, and $\epsilon$ at 3rd order (as $\sqrt{\epsilon}$ enters the integral for the number of efolds). Define

$$
\mathcal{K} \equiv \frac{V_{h}}{V}=\frac{1}{h_{\phi}} \frac{V_{\phi}}{V}=\sqrt{\frac{8}{3}} \frac{\left(1+\frac{\beta_{\lambda}}{4 \lambda}\right) \delta(\delta+1)}{\left(\frac{\delta+1}{6 \xi}+1\right)^{\frac{1}{2}}\left(\delta+1+\frac{\beta_{\xi}}{2 \xi}\right)}
$$

Then the slow roll parameters can be written as

$$
\begin{aligned}
\epsilon & =\frac{1}{2} \mathcal{K}^{2}=\frac{8}{3}\left(1+\frac{\beta_{\lambda}}{4 \lambda}\right) \frac{\delta^{2}(\delta+1)^{2}}{A B^{2}} \\
\eta & =\frac{V_{h h}}{V}=\frac{1}{V} \frac{d}{d h}(\mathcal{K} V)=\mathcal{K}_{h}+\mathcal{K}^{2}=\frac{1}{h_{\phi}} \mathcal{K}_{\phi}+2 \epsilon,
\end{aligned}
$$


where we have defined

$$
A=\left(\frac{\delta+1}{6 \xi}+1\right), \quad B=\left(1+\frac{\beta_{\xi}}{2 \xi}+\delta\right) .
$$

Now $\mathcal{K}_{h}=h_{\phi}^{-1} \mathcal{K}_{\phi}$ with $h_{\phi}=\sqrt{\gamma_{\phi \phi}}$ given in terms of the field space metric (2.5). Explicitly

$$
\mathcal{K}_{h}=\frac{1}{h_{\phi}}\left(\frac{\partial \mathcal{K}}{\partial \delta} \delta_{\phi}+\frac{\partial \mathcal{K}}{\partial f_{j}} \frac{d f_{j}}{d t} \frac{d t}{d \phi}\right)
$$

where $f_{j} \equiv\left\{\lambda, \beta_{\lambda}, \xi, \beta_{\xi}\right\}$ and thus $d f_{j} / d t \equiv\left\{\beta_{\lambda}, \beta_{\lambda}^{\prime}, \beta_{\xi}, \beta_{\xi}^{\prime}\right\}$. The full non-expanded slow roll parameters are given by (B.2) and ${ }^{17}$

$$
\begin{aligned}
\eta= & \frac{V_{h h}}{V}=\frac{4}{3}\left(1+\frac{\beta_{\lambda}}{4 \lambda}\right)\left(\frac{\delta^{2}(\delta+1)^{2}}{A B^{2}}\left(1+\frac{1}{12 \xi} \frac{B}{A}+2\left(1+\frac{\beta_{\lambda}}{4 \lambda}\right)\right)-\frac{\delta(\delta+1)(2 \delta+1)}{A B^{2}}\right) \\
& +\frac{2}{3}\left(\frac{\beta_{\lambda}^{\prime}}{4 \lambda}-\frac{\beta_{\lambda}^{2}}{4 \lambda^{2}}\right) \frac{\delta^{2}(\delta+1)^{2}}{A B^{2}}+O\left(\frac{\beta_{\xi}}{\xi^{2}}, \frac{\beta_{\xi}^{\prime}}{\xi}\right)
\end{aligned}
$$

For $\lambda_{\max }=-\beta_{\lambda} / 4$ or equivalently $F=0, \epsilon$ reduces to zero (extremum of the potential) while $\eta$ reduces to $(3.11)$.

In order to compute the number of efolds we expand $\epsilon$ at third order in $\delta$, which means we need to expand $\mathcal{K}$ at second order in $\delta$,

$$
\mathcal{K} \approx k_{1} \delta+k_{2} \delta^{2}+O\left(\delta^{3}\right)
$$

Then $\epsilon$ is given by $\epsilon=\epsilon_{0} \delta^{2}+\epsilon_{1} \delta^{3}+O\left(\delta^{4}\right)$ with $\epsilon_{0}=k_{1}^{2} / 2$ and $\epsilon_{1}=k_{1} k_{2} . N_{\star}$ becomes

$$
N_{\star}=\int_{\phi_{\text {end }}}^{\phi_{\star}} \frac{1}{\sqrt{2\left(\epsilon_{0} \delta^{2}+\epsilon_{1} \delta^{3}+. .\right)}} h_{\phi} d \phi,
$$

with

$$
\epsilon \approx \frac{1}{2} k_{1}^{2} \delta_{\text {end }}^{2}=1 \quad \Longrightarrow \quad \phi_{\text {end }} \approx\left(\frac{4}{3}\left(1+\frac{1}{6 \xi}\right)\right)^{1 / 4}\left(\frac{F_{\text {end }}}{\xi_{\text {end }}}\right)^{1 / 2} \approx\left(\frac{F_{\text {end }}}{\xi_{\text {end }}}\right)^{1 / 2} .
$$

To understand which terms are important we expand the integrand, i.e. $N_{\star} \equiv \int f$, schematically as $f \sim O(1 / \sqrt{\delta})+O(\sqrt{\delta})+O(\delta)+\ldots$, where $\int O(1 / \sqrt{\delta}) \propto \phi_{\star}^{2} ; \int O(\sqrt{\delta}) \propto \ln \left(\phi_{\star}\right)$; $\int O(\delta) \propto 1 / \xi \phi_{\star}$. The results can be written in term of $\delta_{\star}$ as ${ }^{18}$

$$
N_{\star} \approx a_{1} \frac{1}{\delta_{\star}}+a_{2} \ln \delta_{\star}+\mathcal{C}
$$

with

$$
a_{1}=\frac{3}{4 F_{\star}}, \quad a_{2}=\frac{3}{4 F_{\star}}\left(\frac{1}{1+\frac{1}{6 \xi_{\star}}}+\frac{\beta_{\xi \star}}{2 \xi_{\star}\left(1+\frac{\beta_{\xi \star}}{2 \xi_{\star}}\right)}\right), \quad \mathcal{C}=-\frac{3}{4 F_{\star}} \xi_{\star} \phi_{\text {end }}^{2}+a_{2} \ln \left(\xi_{\star} \phi_{\text {end }}^{2}\right) .
$$

\footnotetext{
${ }^{17}$ For completeness $\frac{1}{3}\left(1+\frac{\beta_{\lambda}}{4 \lambda}\right) \frac{\delta^{2}(\delta+1)^{2}}{A B^{3}}\left[\frac{\beta_{\xi}}{\xi^{2}}\left(\frac{B}{6 A}+\beta_{\xi}\right)-\frac{\beta_{\xi}^{\prime}}{\xi}\right] \equiv O\left(\frac{\beta_{\xi}}{\xi^{2}}, \frac{\beta_{\xi}^{\prime}}{\xi}\right)$.

${ }^{18}$ Following the arguments below (3.14) we neglect the implicit $\phi$ dependence of the couplings and $\beta$ functions.
} 
Now rewrite (B.10) as

$$
\delta_{\star}=\frac{a_{1}}{N_{\star}}+\frac{a_{2} \delta_{\star} \ln \delta_{\star}}{N_{\star}}+\frac{\mathcal{C} \delta_{\star}}{N_{\star}},
$$

which can be solved iteratively. At leading order $\delta_{\star}=a_{1} N_{\star}^{-1}+O\left(N_{\star}^{-2}\right)$ and plugging that back in (B.12) gives

$$
\delta_{\star}=\frac{a_{1}}{N_{\star}}+\frac{a_{2} a_{1}}{N_{\star}^{2}} \ln \left(\frac{a_{1}}{N_{\star}}\right)+\frac{\mathcal{C} a_{1}}{N_{\star}^{2}}+O\left(N_{\star}^{-3}\right) .
$$

Then $\epsilon$ evaluated at horizon exit becomes at second order

$$
\epsilon_{\star} \approx \frac{4}{3}\left(1+\frac{1}{6 \xi_{\star}}\right) F_{\star}^{2} \frac{a_{1}^{2}}{N_{\star}^{2}}=\frac{3}{4} \frac{1}{N_{\star}^{2}}\left(1+\frac{1}{6 \xi_{\star}}\right)
$$

Expanding (B.6) at second order in $\delta$ and using (B.13) (we set also $\xi \gg 1$ for simplicity and we neglect $\left.O\left(\beta_{\xi} / \xi^{2}, \beta_{\xi}^{\prime} / \xi\right)\right)$ we obtain

$$
\begin{aligned}
\eta_{\star} \approx & -\frac{1}{N_{\star}}+\frac{3}{2 N_{\star}^{2}}-\frac{3}{4 N_{\star}^{2}} \frac{1}{F_{\star}}\left(1-\frac{1}{2 F_{\star}}\left(\frac{\beta_{\lambda \star}^{\prime}}{4 \lambda_{\star}}-\frac{\beta_{\lambda \star}^{2}}{4 \lambda_{\star}^{2}}\right)-\ln \left(\frac{\xi_{\text {end }}}{\xi_{\star}} \frac{F_{\star}}{F_{\text {end }}} N_{\star}\right)-\frac{\xi_{\star}}{\xi_{\text {end }}} F_{\text {end }}\right) \\
& +O\left(N_{\star}^{-3}\right) .
\end{aligned}
$$

Therefore the CMB parameters are given by the expressions

$$
\begin{aligned}
n_{s} & =1+2 \eta_{\star}-6 \epsilon_{\star} \\
& \approx 1-\frac{2}{N_{\star}}-\frac{3}{2 N_{\star}^{2}}-\frac{3}{2 N_{\star}^{2} F_{\star}}\left(1-\frac{1}{2 F_{\star}}\left(\frac{\beta_{\lambda \star}^{\prime}}{4 \lambda_{\star}}-\frac{\beta_{\lambda \star}^{2}}{4 \lambda_{\star}^{2}}\right)-\ln \left(\frac{\xi_{\text {end }}}{\xi_{\star}} \frac{F_{\star}}{F_{\text {end }}} N_{\star}\right)-\frac{\xi_{\star}}{\xi_{\text {end }}} F_{\text {end }}\right), \\
r & =16 \epsilon_{\star} \approx \frac{12}{N_{\star}^{2}} .
\end{aligned}
$$

Turning off the running $F_{\star}=1, \beta_{i}=0$, the tree level result are recovered at second order in $N_{\star}^{-1}$, i.e. $n_{s}=1-\frac{2}{N_{\star}}-\frac{3}{N_{\star}^{2}}+\ldots$. The spectral index $n_{s}$ feels the effect of the running only at second order. This dependence goes as $F_{\star}^{-1}$. Note, however, that for values of $F_{\star}$ close to zero the $\delta$ expansion breaks down, and we can no longer trust our analytical results. This is exactly the case where the potential has a maximum and we study the problem numerically.

Open Access. This article is distributed under the terms of the Creative Commons Attribution License (CC-BY 4.0), which permits any use, distribution and reproduction in any medium, provided the original author(s) and source are credited.

\section{References}

[1] R. Fakir and W.G. Unruh, Improvement on cosmological chaotic inflation through nonminimal coupling, Phys. Rev. D 41 (1990) 1783 [INSPIRE].

[2] D.S. Salopek, J.R. Bond and J.M. Bardeen, Designing Density Fluctuation Spectra in Inflation, Phys. Rev. D 40 (1989) 1753 [InSPIRE]. 
[3] F.L. Bezrukov and M. Shaposhnikov, The Standard Model Higgs boson as the inflaton, Phys. Lett. B 659 (2008) 703 [arXiv:0710.3755] [INSPIRE].

[4] F. Bezrukov, The Higgs field as an inflaton, Class. Quant. Grav. 30 (2013) 214001 [arXiv: 1307.0708] [INSPIRE].

[5] Planck collaboration, P.A.R. Ade et al., Planck 2015 results. XX. Constraints on inflation, arXiv: 1502.02114 [INSPIRE].

[6] F. Bezrukov, M. Yu. Kalmykov, B.A. Kniehl and M. Shaposhnikov, Higgs Boson Mass and New Physics, JHEP 10 (2012) 140 [arXiv:1205.2893] [INSPIRE].

[7] G. Degrassi et al., Higgs mass and vacuum stability in the Standard Model at NNLO, JHEP 08 (2012) 098 [arXiv: 1205.6497] [INSPIRE].

[8] V. Branchina and E. Messina, Stability, Higgs Boson Mass and New Physics, Phys. Rev. Lett. 111 (2013) 241801 [arXiv: 1307.5193] [INSPIRE].

[9] V. Branchina and E. Messina, Stability and UV completion of the Standard Model, arXiv: 1507.08812 [INSPIRE].

[10] A. Kobakhidze and A. Spencer-Smith, The Higgs vacuum is unstable, arXiv:1404.4709 [INSPIRE].

[11] A. Spencer-Smith, Higgs Vacuum Stability in a Mass-Dependent Renormalisation Scheme, arXiv: 1405.1975 [INSPIRE].

[12] A.V. Bednyakov, B.A. Kniehl, A.F. Pikelner and O.L. Veretin, Stability of the Electroweak Vacuum: Gauge Independence and Advanced Precision, Phys. Rev. Lett. 115 (2015) 201802 [arXiv: 1507.08833] [INSPIRE].

[13] O. Lebedev, On Stability of the Electroweak Vacuum and the Higgs Portal, Eur. Phys. J. C 72 (2012) 2058 [arXiv:1203.0156] [INSPIRE].

[14] J. Elias-Miro, J.R. Espinosa, G.F. Giudice, H.M. Lee and A. Strumia, Stabilization of the Electroweak Vacuum by a Scalar Threshold Effect, JHEP 06 (2012) 031 [arXiv:1203.0237] [INSPIRE].

[15] L. Basso, O. Fischer and J.J. van Der Bij, A renormalization group analysis of the Hill model and its HEIDI extension, Phys. Lett. B 730 (2014) 326 [arXiv:1309.6086] [INSPIRE].

[16] C.P. Burgess, H.M. Lee and M. Trott, Power-counting and the Validity of the Classical Approximation During Inflation, JHEP 09 (2009) 103 [arXiv:0902.4465] [INSPIRE].

[17] J.L.F. Barbon and J.R. Espinosa, On the Naturalness of Higgs Inflation, Phys. Rev. D 79 (2009) 081302 [arXiv: 0903.0355] [inSPIRE].

[18] C.P. Burgess, H.M. Lee and M. Trott, Comment on Higgs Inflation and Naturalness, JHEP 07 (2010) 007 [arXiv: 1002 . 2730] [INSPIRE].

[19] M.P. Hertzberg, On Inflation with Non-minimal Coupling, JHEP 11 (2010) 023 [arXiv: 1002.2995] [INSPIRE].

[20] F. Bezrukov, A. Magnin, M. Shaposhnikov and S. Sibiryakov, Higgs inflation: consistency and generalisations, JHEP 01 (2011) 016 [arXiv: 1008.5157] [INSPIRE].

[21] S. Ferrara, R. Kallosh, A. Linde, A. Marrani and A. Van Proeyen, Superconformal Symmetry, NMSSM and Inflation, Phys. Rev. D 83 (2011) 025008 [arXiv:1008.2942] [InSPIRE]. 
[22] C.P. Burgess, S.P. Patil and M. Trott, On the Predictiveness of Single-Field Inflationary Models, JHEP 06 (2014) 010 [arXiv:1402.1476] [INSPIRE].

[23] Z.-Z. Xianyu, J. Ren and H.-J. He, Gravitational Interaction of Higgs Boson and Weak Boson Scattering, Phys. Rev. D 88 (2013) 096013 [arXiv: 1305.0251] [INSPIRE].

[24] G.F. Giudice and H.M. Lee, Unitarizing Higgs Inflation, Phys. Lett. B 694 (2011) 294 [arXiv: 1010.1417] [INSPIRE].

[25] J.L.F. Barbon, J.A. Casas, J. Elias-Miro and J.R. Espinosa, Higgs Inflation as a Mirage, JHEP 09 (2015) 027 [arXiv: 1501.02231] [INSPIRE].

[26] U. Aydemir, M.M. Anber and J.F. Donoghue, Self-healing of unitarity in effective field theories and the onset of new physics, Phys. Rev. D 86 (2012) 014025 [arXiv:1203.5153] [INSPIRE].

[27] X. Calmet and R. Casadio, Self-healing of unitarity in Higgs inflation, Phys. Lett. B 734 (2014) 17 [arXiv:1310.7410] [inSPIRE].

[28] I.G. Moss, Covariant one-loop quantum gravity and Higgs inflation, arXiv:1409.2108 [INSPIRE].

[29] J. Ren, Z.-Z. Xianyu and H.-J. He, Higgs Gravitational Interaction, Weak Boson Scattering and Higgs Inflation in Jordan and Einstein Frames, JCAP 06 (2014) 032 [arXiv: 1404.4627] [INSPIRE].

[30] D.P. George, S. Mooij and M. Postma, Quantum corrections in Higgs inflation: the Standard Model case, JCAP 04 (2016) 006 [arXiv: 1508.04660] [INSPIRE].

[31] F. Bezrukov and M. Shaposhnikov, Standard Model Higgs boson mass from inflation: Two loop analysis, JHEP 07 (2009) 089 [arXiv:0904.1537] [INSPIRE].

[32] F. Bezrukov and M. Shaposhnikov, Standard Model Higgs boson mass from inflation: Two loop analysis, JHEP 07 (2009) 089 [arXiv:0904.1537] [INSPIRE].

[33] A. De Simone, M.P. Hertzberg and F. Wilczek, Running Inflation in the Standard Model, Phys. Lett. B 678 (2009) 1 [arXiv:0812.4946] [InSPIRE].

[34] A.O. Barvinsky, A. Yu. Kamenshchik and A.A. Starobinsky, Inflation scenario via the Standard Model Higgs boson and LHC, JCAP 11 (2008) 021 [arXiv:0809.2104] [INSPIRE].

[35] A.O. Barvinsky, A. Yu. Kamenshchik, C. Kiefer, A.A. Starobinsky and C. Steinwachs, Asymptotic freedom in inflationary cosmology with a non-minimally coupled Higgs field, JCAP 12 (2009) 003 [arXiv:0904.1698] [INSPIRE].

[36] A.O. Barvinsky, A. Yu. Kamenshchik, C. Kiefer, A.A. Starobinsky and C.F. Steinwachs, Higgs boson, renormalization group and naturalness in cosmology, Eur. Phys. J. C 72 (2012) 2219 [arXiv: 0910.1041] [INSPIRE].

[37] K. Allison, Higgs xi-inflation for the 125-126 GeV Higgs: a two-loop analysis, JHEP 02 (2014) 040 [arXiv: 1306.6931] [INSPIRE].

[38] D.P. George, S. Mooij and M. Postma, Quantum corrections in Higgs inflation: the real scalar case, JCAP 02 (2014) 024 [arXiv: 1310.2157] [INSPIRE].

[39] D.P. George, S. Mooij and M. Postma, Effective action for the Abelian Higgs model in FLRW, JCAP 11 (2012) 043 [arXiv: 1207.6963] [INSPIRE].

[40] M.P. Hertzberg, Can Inflation be Connected to Low Energy Particle Physics?, JCAP 08 (2012) 008 [arXiv:1110.5650] [INSPIRE]. 
[41] M. Postma and M. Volponi, Equivalence of the Einstein and Jordan frames, Phys. Rev. D 90 (2014) 103516 [arXiv: 1407.6874] [INSPIRE].

[42] E.E. Jenkins, A.V. Manohar and M. Trott, Renormalization Group Evolution of the Standard Model Dimension Six Operators I: Formalism and lambda Dependence, JHEP 10 (2013) 087 [arXiv: 1308.2627] [INSPIRE].

[43] F. Bezrukov, J. Rubio and M. Shaposhnikov, Living beyond the edge: Higgs inflation and vacuum metastability, Phys. Rev. D 92 (2015) 083512 [arXiv:1412.3811] [INSPIRE].

[44] S.R. Coleman and E.J. Weinberg, Radiative Corrections as the Origin of Spontaneous Symmetry Breaking, Phys. Rev. D 7 (1973) 1888 [InSPIRE].

[45] M.P. Hertzberg, Inflation, Symmetry and B-Modes, Phys. Lett. B 745 (2015) 118 [arXiv: 1403.5253] [INSPIRE].

[46] I. Quiros, R. Garcia-Salcedo, J.E.M. Aguilar and T. Matos, The conformal transformation's controversy: what are we missing?, Gen. Rel. Grav. 45 (2013) 489 [arXiv:1108.5857] [INSPIRE].

[47] I. Quiros, R. Garcia-Salcedo and J.E.M. Aguilar, Conformal transformations and the conformal equivalence principle, arXiv:1108.2911 [INSPIRE].

[48] R. Catena, M. Pietroni and L. Scarabello, Einstein and Jordan reconciled: a frame-invariant approach to scalar-tensor cosmology, Phys. Rev. D 76 (2007) 084039 [astro-ph/0604492] [INSPIRE].

[49] J.-O. Gong, J.-c. Hwang, W.-I. Park, M. Sasaki and Y.-S. Song, Conformal invariance of curvature perturbation, JCAP 09 (2011) 023 [arXiv:1107.1840] [INSPIRE].

[50] T. Chiba and M. Yamaguchi, Extended Slow-Roll Conditions and Rapid-Roll Conditions, JCAP 10 (2008) 021 [arXiv:0807.4965] [INSPIRE].

[51] T. Kubota, N. Misumi, W. Naylor and N. Okuda, The Conformal Transformation in General Single Field Inflation with Non-Minimal Coupling, JCAP 02 (2012) 034 [arXiv:1112.5233] [INSPIRE].

[52] J. Weenink and T. Prokopec, Gauge invariant cosmological perturbations for the nonminimally coupled inflaton field, Phys. Rev. D 82 (2010) 123510 [arXiv:1007.2133] [INSPIRE].

[53] T. Prokopec and J. Weenink, Frame independent cosmological perturbations, JCAP 09 (2013) 027 [arXiv: 1304.6737] [INSPIRE].

[54] A. Yu. Kamenshchik and C.F. Steinwachs, Question of quantum equivalence between Jordan frame and Einstein frame, Phys. Rev. D 91 (2015) 084033 [arXiv:1408.5769] [INSPIRE].

[55] L. Senatore and M. Zaldarriaga, On Loops in Inflation, JHEP 12 (2010) 008 [arXiv:0912.2734] [INSPIRE].

[56] D. Buttazzo et al., Investigating the near-criticality of the Higgs boson, JHEP 12 (2013) 089 [arXiv: 1307.3536] [INSPIRE].

[57] F. Bezrukov and M. Shaposhnikov, Higgs inflation at the critical point, Phys. Lett. B 734 (2014) 249 [arXiv: 1403.6078] [INSPIRE].

[58] Y. Hamada, H. Kawai, K.-y. Oda and S.C. Park, Higgs Inflation is Still Alive after the Results from BICEP2, Phys. Rev. Lett. 112 (2014) 241301 [arXiv:1403.5043] [INSPIRE]. 
[59] ATLAS collaboration, Measurements of Higgs boson production and couplings in the four-lepton channel in pp collisions at center-of-mass energies of 7 and $8 \mathrm{TeV}$ with the ATLAS detector, Phys. Rev. D 91 (2015) 012006 [arXiv:1408.5191] [INSPIRE].

[60] J. García-Bellido, D.G. Figueroa and J. Rubio, Preheating in the Standard Model with the Higgs-Inflaton coupled to gravity, Phys. Rev. D 79 (2009) 063531 [arXiv:0812.4624] [INSPIRE].

[61] C.G. Callan Jr., Broken scale invariance in scalar field theory, Phys. Rev. D 2 (1970) 1541 [INSPIRE].

[62] K. Symanzik, Small distance behavior in field theory and power counting, Commun. Math. Phys. 18 (1970) 227 [INSPIRE].

[63] C. Ford, D.R.T. Jones, P.W. Stephenson and M.B. Einhorn, The Effective potential and the renormalization group, Nucl. Phys. B 395 (1993) 17 [hep-lat/9210033] [INSPIRE].

[64] C.M. Fraser, Calculation of Higher Derivative Terms in the One Loop Effective Lagrangian, Z. Phys. C 28 (1985) 101 [INSPIRE].

[65] J. Iliopoulos, C. Itzykson and A. Martin, Functional Methods and Perturbation Theory, Rev. Mod. Phys. 47 (1975) 165 [InSPIRE].

[66] S. Mooij and M. Postma, Goldstone bosons and a dynamical Higgs field, JCAP 09 (2011) 006 [arXiv: 1104.4897] [INSPIRE].

[67] J.R. Espinosa et al., The cosmological Higgstory of the vacuum instability, JHEP 09 (2015) 174 [arXiv: 1505. 04825] [INSPIRE]. 